FAMILY LAW 


\section{FAMILY LAW}

An account of the Law of Domestic Relations in England and Wales in the last quarter of the twentieth century, with some comparisons

Olive M. Stone

LL.B., B.Sc.(Econ.) Ph.D., of Gray's Inn, Barrister Reader in Law to the University of London at the

London School of Economics and Political Science 
(C) Olive M. Stone 1977

Softcover reprint of the hardcover 1st edition 1977 978-0-333-19629-8

All rights reserved. No part of this publication may be reproduced or transmitted, in any form or by any means, without permission.

First published 1977 by

THE MACMILLAN PRESS LTD

London and Basingstoke

Associated companies in New York Dublin

Melbourne Johannesburg and Madras

ISBN 978-0-333-19630-4 ISBN 978-1-349-86147-7 (eBook)

DOI $10.1007 / 978-1-349-86147-7$

This book is sold subject to the standard conditions of the Net Book Agreement.

The paperback edition of this book is sold subject to the condition that it shall not, by way of trade or otherwise, be lent, re-sold, hired out, or otherwise circulated without the publisher's prior consent in any form of binding or cover other than that in which it is published and without a similar condition including this condition being imposed on the subsequent purchaser. 


\section{Contents}

A. Marriage, Family and Household . $\quad . \quad$. $\quad . \quad . \quad . \quad . \quad 1$

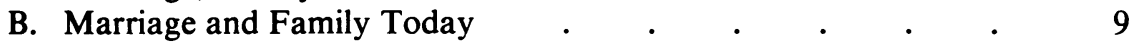

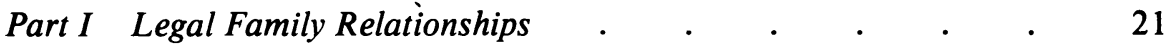

CHAPTER II Before the Ceremony: The Marriage Ceremony: Void and Voidable Marriages and their Effects . . 23

A. Before the Wedding Ceremony . . . . . . $\quad . \quad 23$

(i) Damages for Breach of Promise of Marriage $\quad$. $\quad . \quad$. 23

(ii) The Presumption of Undue Influence $\quad . \quad$. $\quad . \quad$. 25

B. The Marriage Ceremony (or Wedding) . . . . . . $\quad$. 26

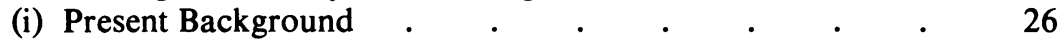

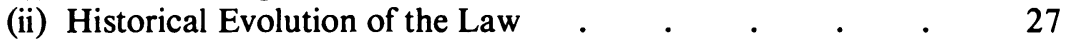

(iii) Preliminaries to Marriage
(a) Civil Preliminaries

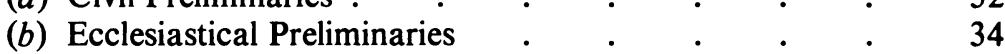

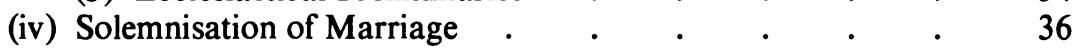

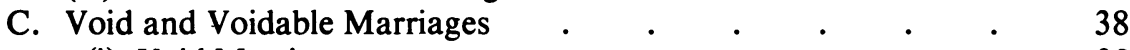

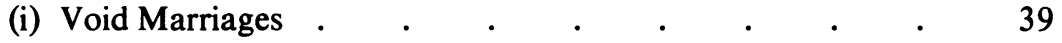

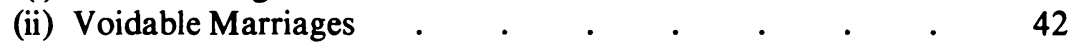

(a) Incapacity to Consummate . . . . . . 42

(b) Wilful Refusal to Consummate $\quad . \quad$. $\quad$. $\quad$. 43 
(d) Mental Disorder $\quad \cdot \quad \cdot \quad \cdot \quad \cdot \quad \cdot \quad \cdot \quad \cdot \quad 44$

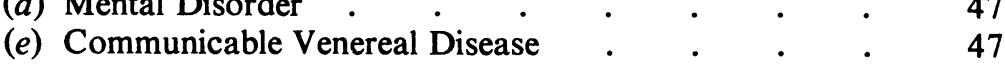

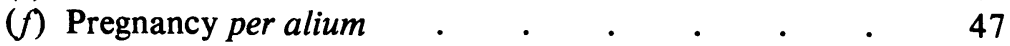

D. Bars to Avoidance of Voidable Marriages $\quad$. $\quad$. $\quad$. $\quad$. 48

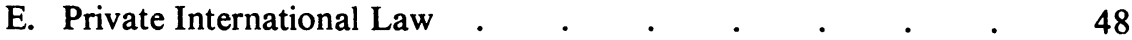

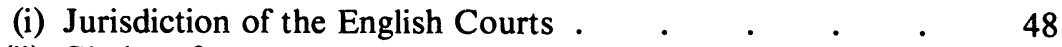

(ii) Choice of Law

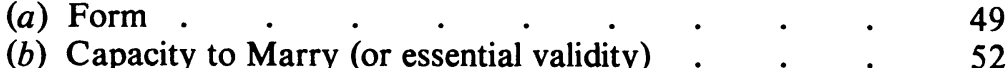

(iii) The Recognition of Polygamous Marrages in English Law. 52

CHAPTER III Personal Legal Relations in the Functioning Family 54

A. Surname . $\quad . \quad . \quad . \quad . \quad . \quad . \quad . \quad . \quad 56$

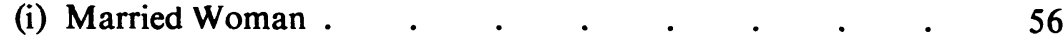

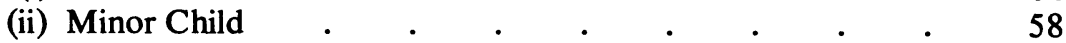

B. Nationality, Citizenship and Patriality $\quad$. $\quad . \quad 5 \quad$. $\quad . \quad 59$

(i) Husband and Wife $\quad . \quad . \quad . \quad . \quad . \quad . \quad . \quad 662$

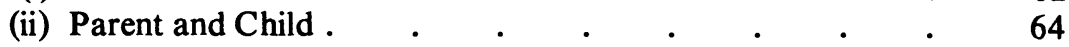

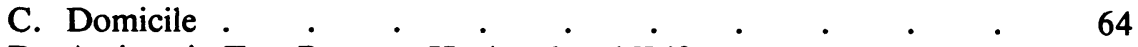

D. Actions in Tort Between Husband and Wife . . . . 65

E. Criminal Injuries Compensation . . . . . . $\quad$. 65

F. Per quod consortium (servitium) amisit . . . . . . . $\quad$. 65

G. Evidence Between Husband and Wife . $\quad$. $\quad$. $\quad . \quad$. 66

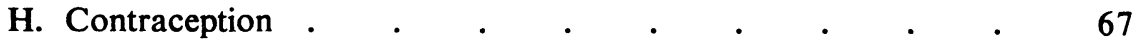

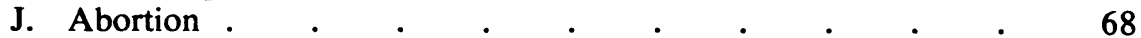

K. Damages for Injury to Unborn Children $\quad$. $\quad$. $\quad$. $\quad$ - 69

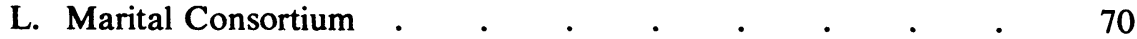

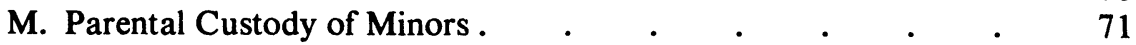

The Reduction and Termination of Parental Authority $\quad$. $\quad$. 73

CHAPTER IV Legal Property and Financial Relations in the Functioning Family . $\quad . \quad$. $\quad$. 75

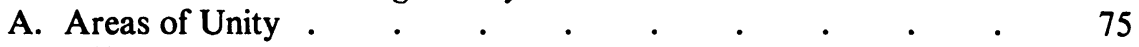

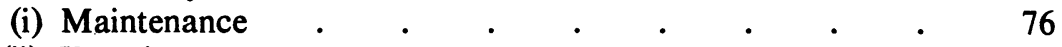

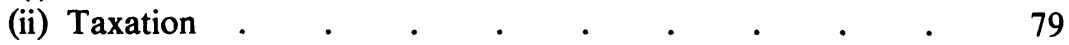

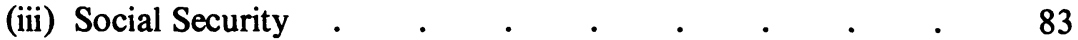

B. Separation of Property - The General Rule and its Mitigation . 87

(i) Transfers of Property Between Spouses to Defeat Creditors' Claims . . . . . . . . . . . 48

(ii) Savings from Housekeeping Allowance $\quad . \quad$. $\quad . \quad$ • 90

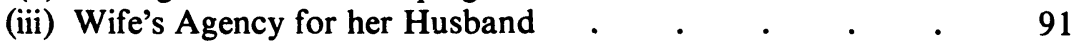

(iv) Other Attempts to Mitigate Separate Property . $\quad$ • $\quad$ • 92 
(v) The Matrimonial Home $\quad 93$

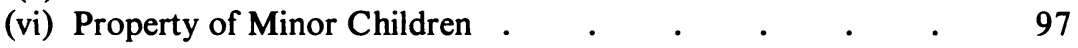

Part II The Disjointed Family . . . . . . . . . . . $\quad$. 103

CHAPTER V Services, Institutions, Courts and their Jurisdictions 105

A. Marital Counselling: Conciliation and Reconciliation Procedures 105

B. Marriage Contracts . . . . . . . . 109

C. Government Conciliation Services. . . . . . . 109

D. Matrimonial Complaints before Magistrates . . . . 110

E. Matrimonial Causes . . . . . . . . . . 116

Divorce fact situations

(i) Adultery and Cohabitation Intolerable $\quad$. $\quad$. $\quad$. $\quad$. 122

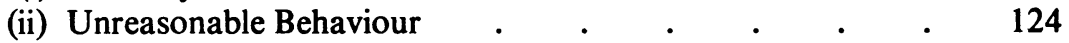

(iii) Two years' Desertion . $\quad . \quad$. $\quad . \quad$. $\quad . \quad$. 126

(iv) Two years' Separation and Respondent's Consent to a Divorce Decree . . . . . . . . . . . 127

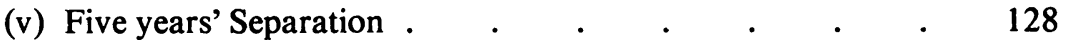

(a) Grave Financial Hardship $\quad . \quad$. $\quad . \quad$. $\quad . \quad 129$

(b) Grave Other Hardship . . . . . . . . $\quad$. 130

F. The Role of Divorce . $\quad . \quad$. $\quad$. $\quad$. $\quad . \quad$. $\quad$. 131

G. No Divorce Within Three Years of Wedding . . . . $\quad$. 132

H. Procedure in Divorce Courts . $\quad . \quad$. $\quad . \quad$. $\quad$.

J. Jurisdiction in Matrimonial Causes, Custody of Children and Ancillary Proceedings

(i) In Domestic Law. $\quad . \quad$. $\quad . \quad$. $\quad . \quad$. $\quad . \quad 137$

(ii) In Private International Law . . . . . . . . $\quad$. 139

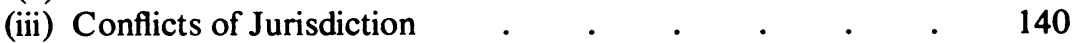

K. Privacy versus Publicity in Family Proceedings . . . 143

CHAPTER VI Dissolution of Marriage by Death. . . . . 147

A. The Law of Intestate Succession . . . . . . . 148

B. No Benefit from Criminal Homicide . . . . . . 151

C. Succession to Statutory Tenancies _ . . . . . . 152

D. The Fatal Accidents Act $\quad . \quad$. $\quad . \quad$. $\quad . \quad$. $\quad . \quad 153$

E. Decree of Presumption of Death and Dissolution of Marriage . 157

F. Family Provision . . . . . . . . $\quad . \quad 157$ The Inheritance (Provision for Family and Dependants) Act 1975

(i) Those Entitled to Apply $\quad . \quad$. $\quad$. $\quad . \quad$. 162

(ii) Orders the Court May Make . $\quad . \quad$. $\quad$. $\quad$. $\quad$. 162

(iii) Property Available for Financial Provision . . . . 164

(iv) Links Between Family Provision and Financial Provision in Matrimonial Causes . . . . . . . . . 167

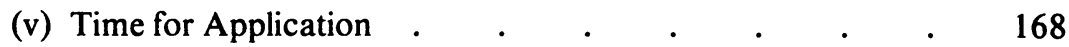

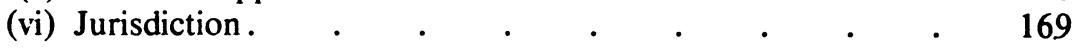


CHAPTER VII The Effects of Separation or Divorce Between Spouses

page

A. Orders in Respect of Title to Property Between Spouses or Former Spouses .

(i) Wachtel v. Wachtel [1973] Fam. 72 C.A.

(ii) Property Claims and Discretionary Allocations

(iii) Property Adjustment Between Cohabitees Not Married to Each Other

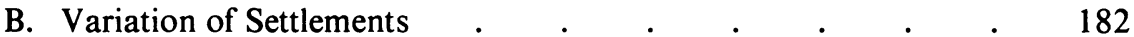

C. Other financial provision under the Matrimonial Causes Act $\quad 183$

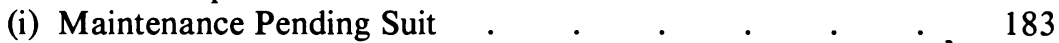

(ii) Financial Provision by Periodical Payments or Lump Sums

(a) Lump Sum Payments . . . . . . . . . .

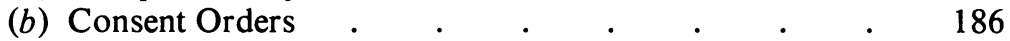

(c) Financial Provision where Supplementary Benefits are paid to one or both parties

D. Maintenance under the Matrimonial Proceedings (Magistrates'

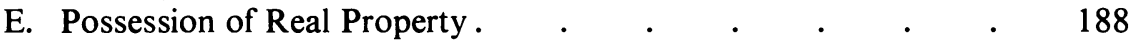

F. Enforcement of Property and Financial Provision Orders . $\quad$. 192

G. International Enforcement of Property and Maintenance Orders

CHAPTER VIII Orders for the Care and Control of Children of Separated Parents . $\quad . \quad . \quad . \quad . \quad . \quad 197$

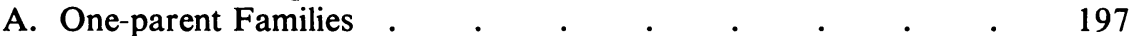

B. Jurisdictions Over Children . $\quad$. $\quad$. $\quad$. $\quad$. $\quad . \quad$. 198

C. General Principles Often Applicable . $\quad$. $\quad$. $\quad$. 208

D. Evidence Receivable in Custody Cases . $\quad$. $\quad$. $\quad . \quad 209$

E. The Individual Jurisdictions

(i) The Inherent Wardship Jurisdiction of the High Court 209

(ii) Habeas Corpus Proceedings . $\quad . \quad$. $\quad . \quad$. $\quad$. 211

(iii) Jurisdiction under the Guardianship of Minors Act 1971 and the Guardianship Act 1973 . . . . .

(iv) Jurisdiction of the Magistrates' Courts under the Matrimonial Proceedings (Magistrates' Courts) Act 1960 and of the Divorce Courts under the Matrimonial Causes Act 1973

(a) Divorce Jurisdiction Contrasted with that on Separation

(b) Children of the Family . . . . . . $\quad . \quad 215$

(vi) Children of Parents not Married to Each Other . . 217

(a) Legitimation and Deemed Legitimacy . . . 217

(b) Illegitimacy . . . . . . . . 219

(vii) Parental Rights over the Ex-nuptial Child . . . 223 
CHAPTER IX Adoption . . . . . . . . . $\quad$. $\quad 225$

A. Historical Outlines of Adoption in England . $\quad$. $\quad . \quad 2 \quad 225$

B. The Balance Between Parental Rights and the Child's Welfare and Changes Effected by the Children Act 1975 . $\quad . \quad$. 230

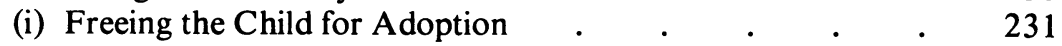

(ii) Court Attendance of Physical Parent not Usually Required 232

(iii) Only Adoption Agencies may Place Children for Adoption 233

(iv) Re-vesting of Parental Rights $\quad . \quad$. $\quad . \quad$. $\quad . \quad 234$

(v) Dispensing with Parental Agreement to Adoption . . $\quad 234$

(vi) Removing a Child from the Home of Prospective Adopters 235

C. Those who May Adopt $\quad . \quad$. $\quad . \quad$. $\quad . \quad 236$

D. Orders for Less Than Full Adoption ․ . $\quad$. $\quad$ • $\quad$. 237

E. Adoption Orders in Private International Law. . . . $\quad$. 238

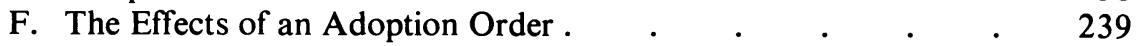

G. Integration of Adoption with Child Welfare Provisions . $\quad$ - 241

CHAPTER X Failure of Parental Duties and Public ResponsibilityChildren in the Care of Public Authorities . .

A. Outlines of the Development of Public Care for Children since

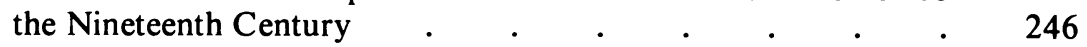

B. Children in Care - The Children Acts 1948-75 . . $\quad$. 247

C. The Children and Young Persons Acts 1933-69, as amended . 256 


\section{Preface}

This book is based on the lectures on the Law of Domestic Relations for students for the Bachelor of Laws degree that I have delivered for some years at the London School of Economics and Political Science. Much of it was written during special leave in the law libraries of Monash University, Australia and the University of Singapore, and my thanks are due to the staff of these and other law libraries for their ever-ready help and co-operation. I thank also those, especially Mss Jacquie Fleming and Mary Choo, who produced a fair typescript. In the later stages my colleague Mr David Bradley was good enough to read the entire script and make valuable suggestions for improvement. Any views expressed are, of course, entirely my own.

Although this book is intended primarily for law students both in England and Wales and overseas, I hope that others will find it a useful statement of the principal problems and areas of interest in family law today.

The law is given as at 20 August 1976, but some decisions reported to 22 October have been briefly noted. 


\section{Table of Statutes}

\section{(1) UNITED KINGDOM STATUTES}

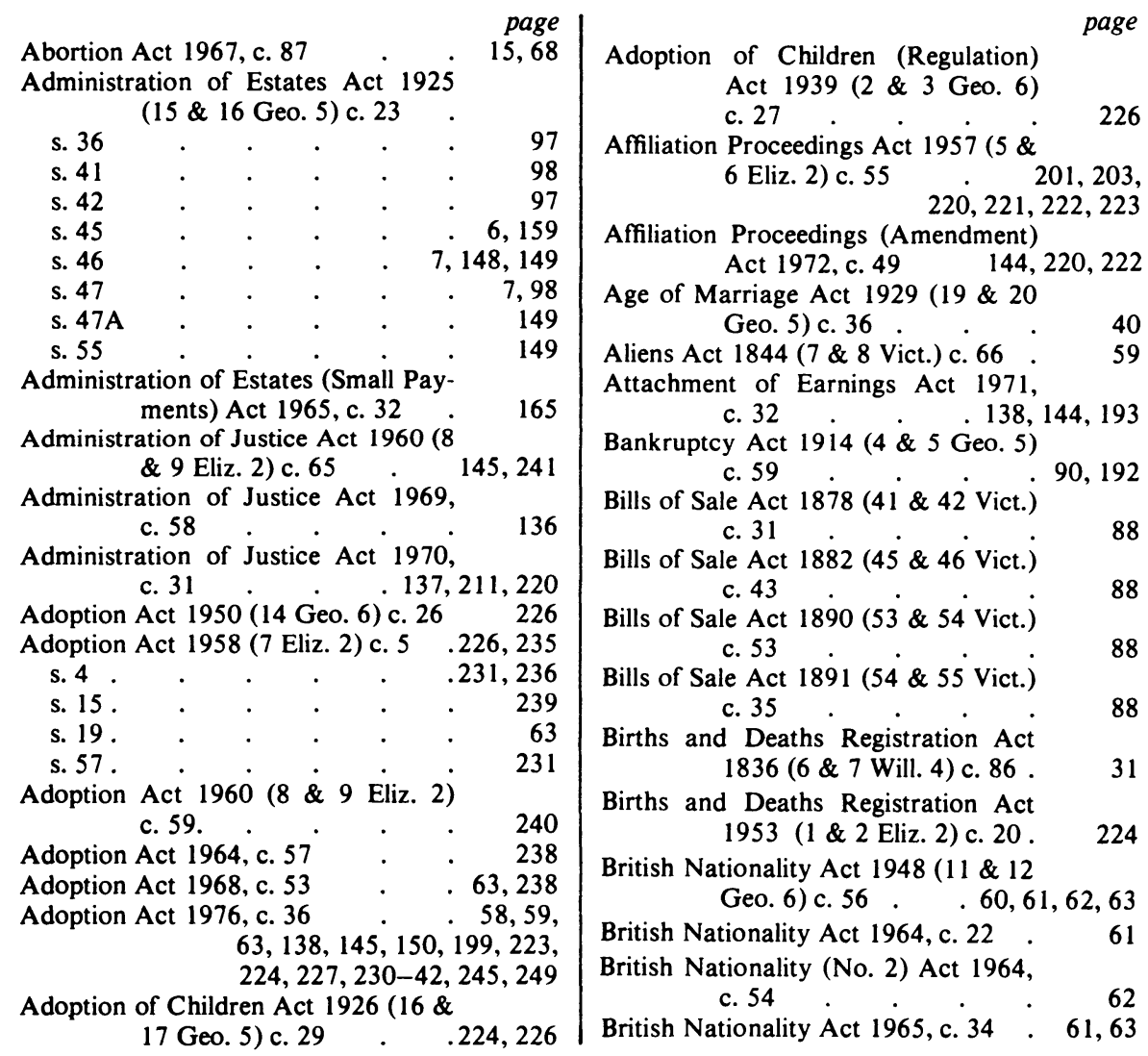


British Nationality and Status of Aliens Act 1914 (4 \& 5 Geo. 5) c. 17 . . .

British Nationality and Status of Aliens Act 1933 (23 \& 24 Geo. 5) c. 49

Carriage by Air Act 1961 (9 \& 10 Eliz. 2) c. 27

Child Benefit Act 1975, c. $61 \quad 75,83,85$

Children Act (An Act for the Prevention of Cruelty to and protection of children) 1889 (52 \& 53 Vict.) c. 44

Children Act 1908 (8 Edw. 7) c. $67 \quad . \quad 246,256$

Children Act 1948 (11 \& 12 Geo. 6) c. $43 \quad 138,145,210,245,247$

s. 1 . . $210,215,247-8,251,252$

s. 2 . $\quad . \quad$. $\quad . \quad$. 248-9, 252

s. 12 .

252

s. 26 .

223

Children Act $1958^{\circ}$ (6 \& 7 Eliz. 2)

c. $65 \quad 138,145,226,245,255$

Children Act 1972, c. 44 .

Children Act 1975, c. 72 .

100

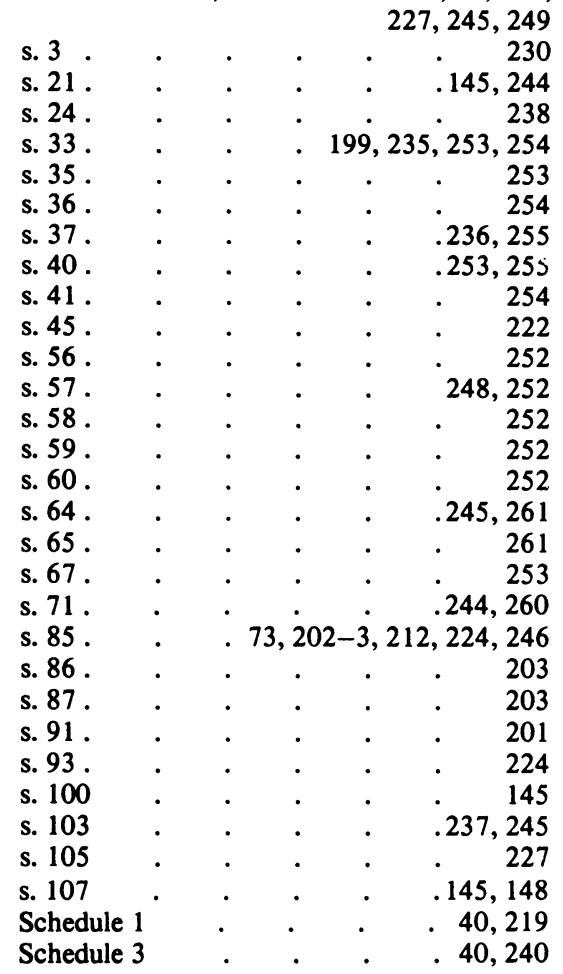

Children and Young Persons Act 1933 (23 \& 24 Geo. 5) c. $12 \quad . \quad 99,138,145,245$, 247, 256, 261

Children and Young Persons Act 1963 , c. $37 \quad$. $\quad 99,100,138$, 210, 248, 257

Children and Young Persons Act 1969, c. $54 \quad$. $138,145,244,249$ 256-62
s. 1 $247-8,258-9,261$

s. 3 . 259

s. 4 244

s. 5 244

s. 9 258

s. 11 . 258

s. 13 . 258

s. 32 . 245

s. 72 . 210

Civil Evidence Act 1968, c. 64 . . 66, 123

Coal Mining (Subsidence) Act 1957 (5 \& 6 Eliz. 2) c. 59 .

Commonwealth Immigrants Act 1962 (10 \& 11 Eliz. 2) c. 21 .

Congenital Disabilities (Civil Liability) Act 1976, c. 28

Council of Merton $1235-6$ (20 Hen. 3) . . 14, 28, 217

County Courts Act 1959 (7 \& 8 Eliz. 2) c. 22 . $.136,192$

Court of Probate Act 1857 (20 \& 21 Vict.) c. $77 \quad$. $\quad$. $\quad 27$

Courts Act 1971, c. 23 . 136, 139, 144, 220

Criminal Evidence Act 1898 (61 \& 62 Vict.) c. 36 .

Custody of Children Act 1891 (54 \& 55 Vict.) c. 3

Custody of Infants Act 1873 (36 \& 37 Vict.) c. 12 .

13,72

Deceased Brother's Widow's Marriage Act 1921 (11 \& 12 Geo. 5) c. 24

Deceased Wife's Sister's Marriage Act 1907 (7 Edw. 7) c. 47 .

Divorce Reform Act 1969, c. 55 . 5, 107, $108,117,122,128$

Divorce (Scotland) Act 1976, c. 39 140

Domestic and Appellate Proceedings Act 1968, c. 63 $143,146,220$

Domestic Violence and Matrimonial Proceedings Act 1976, c.50 $71,137,140,189,191$

Domicile and Matrimonial Proceedings Act 1973, c. 45 . 18, 48, 


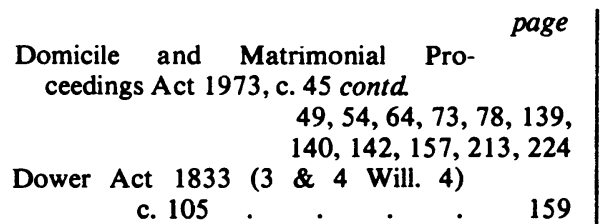

Ecclesiastical Licences Act 1533 . 35

Education Act 1944 (7 \& 8 Geo. 6)

c. $31 \quad$. $\quad 18,99,210,259$

Education (Miscellaneous Provisions) Act 1948 (11 \& 12 Geo. 6) c. 40

Elementary Education Act $1870(33$ \& 34 Vict.) c. 75

Employment of Children Act 1973, c. 24 ,

Employment of Women, Young Persons and Children Act 1920 (10 \& 11 Geo. 5) c. 65

Employment Protection Act 1975, c. $71 \quad$. $\quad 83,84-5$

Equal Pay Act 1970, c. $41 \quad$. $\quad$. 12, 173

Factories Act 1802 (43 Geo. 3) c. 73 . .

Factories Act 1833 (3 \& 4 Will. 4) c. 103 . . 15,246

Factories Act 1961 (9 \& 10 Eliz. 2) c. 34 Part VI

Family Allowances Act 1945 (8 \& 9 Geo. 6) c. 41 .

Family Allowances Act 1965, c. 53 .

Family Income Supplement Act 1970, c. 55

Family Law Reform Act 1969, c. 46 .
s. 1 .
$13,17,4$
s. 3 .
s. 7 .
s. 8 .
s. 9 .
s. 11 .
s. $14-19$
s. 18 .
s. 20 .
s. 21 .
s. 23 .
s. 26 .
s. 27 .
s. 32 .
Sched. 1
Family Provision Act 1966, c. 35
Fatal Accidents Act 1846 (9 \& 10 Vict.) c. 93

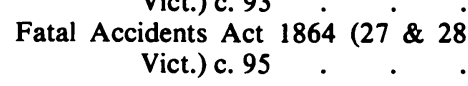

$17,148,151,189,219,220$

. $. \quad 160,219$

. $\quad 219$

219

41,73

160,161

35

(

99

219

219 224

33 148 ,

\section{Fin}

Finance Act 1971, c. 68.
Finance Act 1972 c. 41.

Finance Act 1974, c. 30 . $\quad$. $\quad$. 82

Finance Act 1975, c. $7 \quad$. $\quad . \quad$. 82

Finance (No. 2) Act, 1975, c. $45 \quad 79$

Finance Act 1976, c. 40 . . 79, 81,82

Foreign Marriages Act 1892 (55 \& 56 Vict.) c. $23 \quad$. . 50

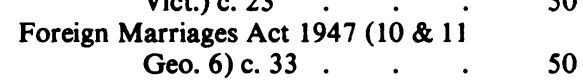

Gas Act 1965, c. 36 • $\quad$ - 153

Guardianship Act 1973, c. 29 . 13, 72, 73, $98,113,115,138,144$, $145,199,200,202,203$, $208,212,213$

Guardianship of Infants Act 1886 (49

$$
\text { \& } 50 \text { Vict.) c. } 27 \text {. 13, 29, } 71
$$

Guardianship of Infants Act 1925 (15 \& 16 Geo. 5) c. 45 . 72, 73, 211

Guardianship of Minors Act 1971,

c. $3 \quad$. $15,65,72,73,98$, $113,114,115,138,144,145$, $185,198-9,200,201,203$, $211,212,213,224,232,254$

Immigration Act 1971, c. $77 \quad .60,61,62,63$

Income and Corporation Taxes Act 1970, c. $10 \quad$. $79,80,81,82,97$

Industrial Injuries and Diseases (Old Cases) Act 1975, c. 16 .

Infants Custody Act (Talfourd's Act) 1839 (2 \& 3 Vict.) c. 54 . 13, 29

Inheritance (Family Provision) Act 1938 (1 \& 2 Geo. 6) c. 45 . 17, 24, $149,159,16 \mathrm{i}, 163$

Inheritance (Provision for Family and Dependants) Act 1975, c. $63 \quad . \quad 17,96,138,161-9$

Intestates Estates Act 1952 (15 \& 16 Geo. 6 and 1 Eliz. 2) c. $64 \quad . \quad .148,149,159$

Judicature, Supreme Court of, (Consolidation) Act 1925 (15 \& 16 Geo. 5) c. $49 \quad$. . .136, 192

Judical Proceedings (Regulation of Reports) Act 1926 (16 \& 17 Geo. 5) c. 61 . 
Law of Property Act 1925 (15 \& 16 Geo. 5) c. 20
s. 1(6)
ss. $23-33$
s. 30 .
s. 40 .
s. 52 .
s. 53 .
s. 172
s. 175

Law Reform (Contributory Negligence) Act 1945 (8 \& 9 Geo. 6) c. 28

Law Reform (Husband and Wife) Act 1962 (10 \& 11 Eliz. 2) c. 48

Law Reform (Limitation of Actions) 1954 (2 \& 3 Eliz. 2) c. 36

Law Reform (Married Women and Tortfeasors) Act 1935 (25 \& 26 Geo. 5) c. 30

Law Reform (Miscellaneous Provisions) Act 1934 (24 \& 25

Geo. 5) c. 41 . $.155,156$

Law Reform (Miscellaneous Provisions) Act 1949 (12, 13 \& 14 Geo. 6) c. 100 .

64,67 78, 183, 209, 218

Law Reform (Miscellaneous Provisions) Act 1970, c. 33

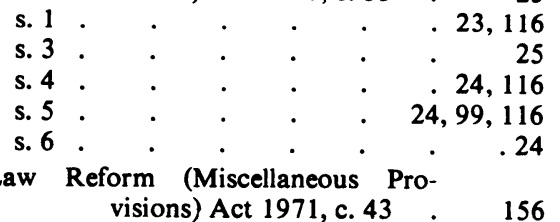

Legal Aid Act 1974, c. 4 . $.107,261$

Legitimacy Act 1926 (16 \& 17 Geo. 5) c. 60 . $28,217,218,219$

Legitimacy Act 1959 (7 \& 8 Eliz. 2) c. 73

$15,144,218,219$, $220,221,224$

Legitimacy Act 1976, c. 31 . 150, 218,219

Legitimacy Declaration Act 1858 (21 \& 22 Vict.) c. $93 \quad$. 201

Licensing Act 1964, c. 26 s. 168

Limitation Act 1975 , c. 54 . . 74, 100

Local Authority Social Services Act 1970, c. $42 \quad$. $\quad . \quad 12,244,258$

Local Government Act 1972, c. 70 . 113

London Government Act 1724

(2 Geo. I) c. 18
Magistrates' Courts Act 1952 (15 \& 16 Geo. 6 \& 1 Eliz. 2)

c. $55 \quad 71,106,110,112,115$,

page

(1)

Magna Carta 1215 (17 John) ch. 18 144

Maintenance Agreements Act 1957

(5 \& 6 Eliz. 2) c. 35 .
Maintenance Orders (Facilities for

157 108 Enforcement) Act 1920 (10 \& 11 Geo. 5) c. 33 . .

Maintenance Orders Act 1950 (14 Geo. 6) c. 37 .

Maintenance Orders Act 1958 (6 \& 7 Eliz. 2) c. $39 \quad$. $\quad$ 138, 187, 192

Maintenance Orders Act 1968, c. 36 .112, 220

Maintenance Orders (Reciprocal Enforcement) Act 1972, c. 18 . $138,144,194-5$

Maritime Conventions Act 1911 (1 \& 2 Geo. 5) c. 57 . .

Marriage Act 1753 (26 Geo. 2) c. 33 (Lord Hardwicke's Act) . 13, 26, $29,30,51$

Marriage Act 1835 (5 \& 6 Will. 4) $\begin{array}{lllll}\text { c. } 54 & \text {. } & & \end{array}$

Marriage Act 1836 (6 \& 7 Will. 4) c. 85 . . .

Marriage Act 1860 (23 \& 24 Vict.)

Marriage Act $1898^{\circ}$ (61 \& $62^{\circ}$ Vict.)

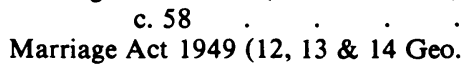

Marriage Act $1949(12,13 \& 14$ Geo.
6) c. 76 .
s. 3
s. 5 .
$33,144,145$
ss. $6-14$
33
ss. $15-16$
34,35
s. 17 .
29, 35
ss. 20-1
33
s. 22 .
36
s. 25 .
37
31,34
s. 26 .
s. 27 .
s. 28 .
s. 29 .
s. 30 .
s. 31 .
s. 32 .
s. 40 .
s. 44 .
ss. $45-6$
ss. $48-9$
s. 75 .
s. 78 .
33, 36, 37
32,33
33
33
33
33
33
32
37
36,37
34
36,37
40
Sched. 1
. 40,240

32-37, 39, 109 
Table of Statutes $\quad$ xvii

Marriage Act 1949 (Amendment) Act 1954 (2 \& 3 Eliz. 2) c. 47.

Marriage Acts Amendment Act 1958 (6 \& 7 Eliz. 2) c. 29 .

Marriage (Enabling) Act 1960 (8 \& 9 Eliz. 2) c. 29

Marriage (Prohibited Degrees of Relationship) Act 1931 (21 \& 22 Geo. 5) c. 31

Marriage (Registrar General's Licence) Act 1970, c. 34

Marriage (Scotland) Act 1939 .

Marriage (Secretaries of Synagogues) Act 1959 (7 \& 8 Eliz. 2) c. 13

Marriage (Wales and Monmouthshire) Act 1962 (10 \& 11 Eliz. 2) c. 32

Marriage with Foreigners Act 1906 (6 Edw. 7), c. 40

Married Women's Property Act 1882 (45 \& 46 Vict.) c. 75

.

s. 10 .

s. 12 .

s. 17 .

$$
\begin{aligned}
& \begin{array}{llll}
. & & & 75 \\
& & 8 & 88
\end{array} \\
& \text {. } . \quad \cdot \quad \text {. } 65
\end{aligned}
$$

Married Women's Property Act 1964

$$
\text { c. } 19
$$

Matrimonial Causes Act 1857 (20 \& 21 Vict.) c. 85

Matrimonial Causes Act 1859 (22 \& 23 Vict.) c. 61 .

Matrimonial Causes Act 1878 (41 \&

42 Vict.) c. 19 . $\quad 77,78,111$

Matrimonial Causes Act 1937 (1

Edw. 8 \& 1 Geo. 6) c. 57 . 4, 38,

$43,47,64,157,218$

Matrimonial Causes Act 1963, c. 45 . 108, 185

Matrimonial Causes Act 1965, c. 72 . 47, 67, $134,151,159,161,220$

Matrimonial Causes Act 1967, c. 56 . 116-17,

183

Matrimonial Causes Act 1973, c. 18 . 25, 117 ,

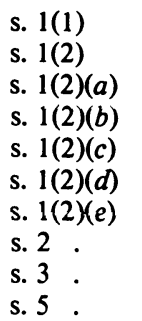
$133,138,139,199,210$

5,117

117

$117,122-4,137$

$114,117,124-5$

- $117,126,137$

- $25,122,137$

$25,122,128-31,137$

$108,122,124,126,127$

132-3 page

Matrimonial Causes Act 1973, c. 18 contd.

s. 6 .

s. 10 .

108,170

$127,128,129$

s. 11 .

s. 12 .

s. 13 .

s. 15 .

$32,36,39,42,53$

$.4,42,48$

. $43,44,47,48$

s. 16 .

133

s. 18 .

s. 19 .

s. 21 .

s. 22 .

s. 23 .

$39,42,48,218$

$.112,151$

$41,48,139,157$

. $135,171,183$

. $\quad .135,183$

$95,135,143,167$

s. 24 .

$171,179,181,183,185$

$96,135,171,172$,

182-3, 192

s. 25 . $\quad$. $\quad$. $\quad .135,171,174$,

$176,183,216$

. $135,171,183$

$78,95,135,140$,

$142,171,183-4$

$135,159,177,183$

. $135,171,192$

s. 30 .

186

s. 32 .

s. 33 .

$135,171,183$

s. 34 .

s. 35 .

s. 36 .

s. 37 .

s. 39 .

s. 41 .

$108,138,168,171$

$.108,138$

$108,138,168,171$

$.135,192$

. 90,171

19, 133-4, 199, 200, 210,

$213,214,253,254$

s. 42 . $\quad$. $\quad$. $59,203,210,214$

s. 43 .

203, 210, 215

s. 44 .

$200,203,215$

s. 45 .

$145,201,220$

s. 46 (repealed) . . . . 48,64, 139

s. 47 .

41,53

s. 48 .

67,144

S. 52 . Patrimonial Causes (Property and
4al

Maintenance) Act 1958 (6

\& 7 Eliz. 2) c. 35

159

Matrimonial Homes Act 1967 , c. 75 . 93, 94, $138,171,178,189-90,191$

Matrimonial
Mroceedings
and

Property Act 1970 , c. $45.113,172$,

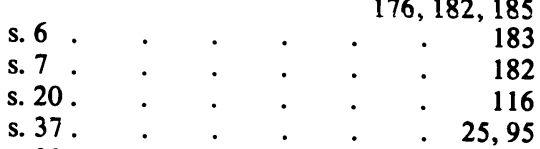

s. 38 . . . . . 93, 171,190

s. 39 .

s. 40 .

$.148,150$ 
Matrimonial Proceedings and Property Act 1970, c. 45 contd.

s. 41 .

Matrimonial Proceedings (Magistrates' Courts) Act 1960 (8 \& 9 Eliz. 2) c. 48 . 110, 115, 138, $142,171,213$

s. 1

$.111,115$

s. 2 .

s. 4 .

s. 8 .

s. 9 .

s. 10 .

s. 12 .

s. 13 .

s. 16 .

Matrimonial Proceedings (Polygamous Marriages) Act 1972 c. 38

Mental Deficiency Acts 1913-1938 (3 \& 4 Geo. 5$)$ c. $28-(1 \&$ 2 Geo. 6) c. 43

Mental Health Act 1959 (7 \& 8 Eliz. 2) c. 72

Mines and Quarries Act 1954, Part VIII (2 \& 3 Eliz. 2) c. 70 .

Ministry of Social Security Act 1966, see Supplementary Benefit Act 1966

Misrepresentation Act 1967, c. 7

National Assistance Act 1948 (11 \& 12 Geo. 6) c. 29 .

National Health Service (Family Planning) Amendment Act 1967 , c. 39

National Health Service (Family Planning) Act 1972, c. 72

National Health Service Reorganisation Act 1973 , c. 32 .

Nuclear Installations Act 1965, c. 57

Nullity of Marriage Act 1971, c. 44 . $38,42,43,44,48,53$

Offences Against the Person Act 1861 (24 \& 25 Vict.) c. 100 5,111

Perjury Act 1911 (1\& 2 Geo. 5) c. 6

Places of Worship Registration Act 1855 (18\& 19 Vict.)c. 81 .

Poor Law Act 1576 (10 Eliz. 1) c. 3 .

Poor Law Amendment Act 1844 ( 7 \& 8 Vict.) c. 101 .

$$
\text { S. } 2
$$

Poor Law Amendment Act 1868 (31 \& 32 Vict.) c. 122

41,53

42,47

111 31 14,223 14,223 s. 37 .
Prevention of Cruelty to and Protection of Children 1889: see Children Act 1889

Prevention of Cruelty to Children (Amendment) Act 1894 (57 \& 58 Vict.) c. $27 \quad$. 246

Race Relations Act 1968, c. $71 \quad$. 250

Recognition of Divorces and Legal Separations Act 1971, c. $53 \quad$. 49, 134, 141, 142

Redundancy Payments Act 1965, c. 62

Registration of Business Names Act $1916.5 . \quad . \quad 56$

Registration Service Act 1953 (1 \& 2 Eliz. 2) c. $37 \quad$. $\quad$. $\quad$. $\quad 32$

Rent Act 1968, c. $23 \quad$. $\quad$. $\quad 152,189$

Royal Marriages Act 1772 (12 Geo. 3) c. 11 . $\quad 31$

Sex Discrimination Act 1975, c. 65 . 12

Sexual Offences Act 1956 (4 \& 5 Eliz. 2) c. $69 \quad$. 3, 16, 67, 99, 245 ,

Shops Act 1950 (14 Geo. 6) c. 28 - 99

Social Security Act 1973, c. 38 . 83,86
Social Security Act 1975, c. $14 . \quad 83,84$

$\begin{array}{rl}85 & 85,\end{array}$

Social Security Benefits Act 1975, c. 11.83

Social Security (Consequential Provisions) Act 1975 , ch. 18 . 83

Social Security Pensions Act 1975, c. 60

Social Work (Scotland) Act 1968, c. 49

Statute Law Repeals Act 1969, ch. 52

Statute of Distributions 1670 (22 \& 23 Chas. 2) c. $10 \quad . \quad .148,158$

Statute of Uses 1535 (27 Hen. 8) c. 10 . . .

Summary Procedure (Domestic Proceedings) Act 1937 (1 Edw. 8 \& 1 Geo. 6) c. 58 .

Supplementary Benefits Act 1966, c. 20 '

Supreme Court of Judicature (Con-
solidation) Act 1925: see Judicature, Supreme Court of. (Consolidation) Act

Taxes Act 1970: see Income and Corporation Taxes Act, 1970

Tenures Abolition Act 1660 (12 Chas. 2) c. 24 . $\quad 13,72,98$

Theft Act 1968, c. $60 \quad$. $\quad$. $\quad 67$

Trade Union and Labour Relations $\begin{array}{lll}\text { Act } 1974, \text { c. } 52 \quad \text {. } & \text {. } & 84\end{array}$ 
Table of Statutes $\quad \mathrm{xix}$

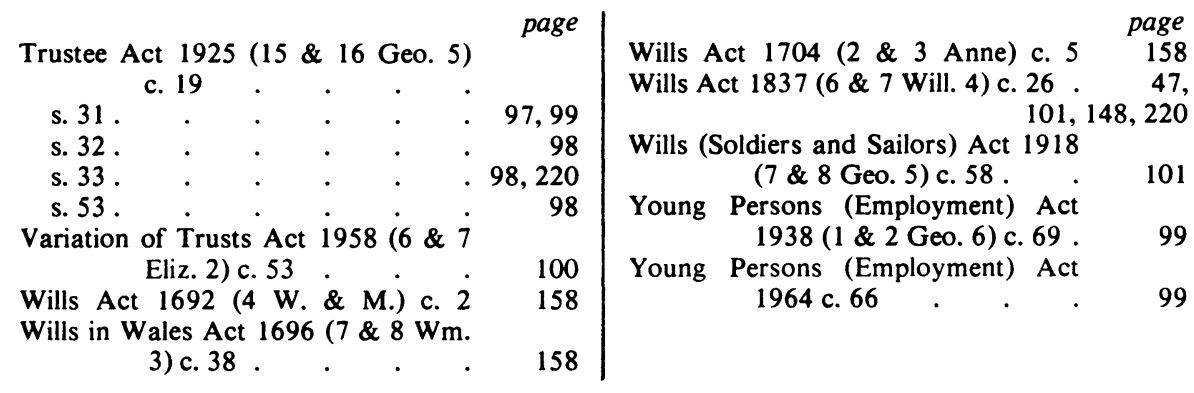

\section{(2) EUROPEAN COMMUNITIES STATUTES}

Treaty of Rome . . . 60, 195-6

\section{(3) OVERSEAS STATUTES}

Australian Matrimonial Causes Act Australian Family Law Act 1975, 106 No. 53 . . 39, 106, 109, 116, $131,133,136,198,212$

China, People's Republic of, Marriage Law 1950

Code Civil Français

German Federal Republic B.G.B. Malaysia: Law Reform (Marriage and Divorce) Bill, 1975

New Zealand Family Protection Act 1900 (64 Vict. 20)
Pakistan Muslim Laws Ordinance (viii 1961). . . 7,62 Singapore: Women's Charter 1961 . 8

Code Civil Suisse . . . . . 23, 24

U.S.A.:

California Civil Code, ss. 36, 37 . 99

New York: Domestic Relations Law . . . . 106

U.S.S.R.: Fundamental Principles of Soviet Family Law (1968)

art. $9 . \quad . \quad . \quad 27,224$ 


\section{Table of Statutory Instruments}

Adoption of Children (Regulation) Act 1939 (Commencement) Order No. 378 of 1943

Adoption (High Court) Rules S.I. No. 1645 of 1976

Adoption (County Court) Rules, S.I. No. 1644 of $1976 \quad . \quad .145,227$

Blood Tests (Evidence of Paternity) Regulations No. 1861 of 1971 .

County Court Rules, S.R. \& O. 1936, No. 626.

Divorce County Courts Order No. 314 of 1968 .

Divorce County Courts No. 1954 of 1971

Divorce County Courts (Amendment) Order No. 1746 of 1972

Divorce County Courts (Amendment) No. 1278 of 1973

Divorce County Courts (Amendment) Order No. 1004 of 1974

Employment Protection Act (Commencement No. 4) Order No. 530 of 1976

Enrolment of Deeds (Change of Name) Regulations No. 316 of 1949 as amended by No. 377 of 1951

Family Income Supplement (Commencement) Order No. 225 of 1971

Family Income Supplement (General) Regulations, Nos. 226, 227, 622 and 702 of 1971 ; Nos. 14, 135 and 1282 of 1972; and Nos. 177 and 1362 of $1973 ; 59$ and 905

Family Income Supplement (General)

page

Regulations, contd. of $1974 ; 1360$ of $1975 ; 289$ and 806 of 1976

Family Law Reform Act 1969 (Commencement No. 2) Order No. 1857 of 1971 .

Family Provision (Intestate Succession) Order No. 916 of 1972

Guardianship Act 1973 (Commencement) Order No. 695 of 1974 . 13, 72,

Immigration Act 1971 (Commencement) Order No. 1514 of 1972 .

Local Authority Social Services Act 1970

(Commencement No. 1) Order No. 1143 of $1970 \quad$. $\quad 258$

(Commencement No. 2) Order No. 1813 of 1970

(Commencement No. 3) Order No. 1221 of 1971

Local Government Act 1972 (Commencement No. 1) (England) Order No. 373 of 1973 .

Local Government Act 1972 (Commencement No. 2) (Wales) Order No. 375 of 1973 .

Magistrates' courts (Adoption) Rules, S.I. No. 1768 of $1976 \quad . \quad .145,227$

Magistrates' Courts (Blood Tests) Rules No. 1991 of 1971 .

Maintenance Orders (Reciprocal Enforcement) Act 1972, Order No. 517 of 1974 
Matrimonial Causes Act 1967 (Commencement) Order No. 228 of 1968

Matrimonial Causes (Decree Absolute) General Order of 20.7.72 (S.I.s 1972 Part II, p. 4109)

Matrimonial Causes (Decree Absolute) General Order of 15.3.73 (S.I.s 1972 Part I, p. 2634)

Matrimonial Causes Rules 1973 No. 2016 of 1973 as amended by Nos. 1383 and 2168 of 1974 , No. 1359 of 1975 and No. 607 of 1976 . 19, 107, 117, 123,127, 133, 134. 135, 137, 138, 143, $170,214,215,216$
Matrimonial Causes (Special Pro-

page cedure Extension) Order No. 1359 of 1975 . $137,144,186$

Raising of School Leaving Age Order No. 444 of 1972

18,73

Recovery abroad of Maintenance (Convention Countries) Order No. 423 of 1975

Registration of Births, Deaths and Marriages Regulations No. 2049 of 1968 .

Rules of Supreme Court Order 99 No. 337 of 1976

Secretary of State for Social Services Order No. 1699 of 1968

Social Security (Reciprocal Agreements) Order No. 225 of 1976 


\section{Table of Cases}

-Indicates a temporary reference.

A.v. A. (Family: Unborn Child [1974] Fam. 6 .

In re A. (An Infant) [1963] 1 W.L.R. 231

A.W. v. E. The Times 29.11 .74

In re A.B. (An Infant) [1954] 2 Q.B. 385

In re A.H. (Infants) [1963] Ch. 232

Abercrombie v. Abercrombie [1943] 2 All E.R. 465

Adams v. Adams [1965] 109 Sol. Jo. 899

Ackerman v. Ackerman [1972] Fam. 225 C.A.

Adams v. Adams [1965] 109 Sol. J. 899

In re Adoption Application $41 / 6 i$ [1963] Ch. 315 C.A. .

In re Adoption Application 41/61 (No. 2) [1964] Ch. 48 . 19,206 ,

231,232

In re Agar-Ellis (1883) 24 Ch.D. $317.13,55,71,207,224$

Ex parte Akhtar, Reg. v. Secretary of State for Home Dept [1975] I W.L.R. 1717

Aldrich v. Attorney-General [1968] P. 281 .

Reg. v. Algar [1954] 1 Q.B. 279

Alhaji Mohamed v. Knott [1969] 1 Q.B. 1

Allen v. Allen [1974] 1 W.L.R. 1171; 3 All E.R. 385 C.A.

Ally v. Ally, The Times 24.8.71

- The Ampthill Peerage [1976] 2 W.L.R. 777 .

181

186 page

216

230

209

251

210

108

136

184

136

232

Att

Andrews v. Andrews [1974] 3 All E.R. $643 . \quad . \quad . \quad . \quad 124,130$ In re Andrews deceased [1955] 1 W.L.R. 1105; 3 All E.R. 248 . 160 In re Andrews [1968] Ch. 665 . . 209 R. v. Andrews (1873) L.R. 8 Q.B. 153

207 Andrews v. Salt (1873) 8 Ch. App. 622.207

Antoniadi v. Smith [1901] 2 K.B. $589 \quad 88$

In re Application No. 4 of 1974, The Times 1.5.75 C.A. . . . 234

Apt v. Apt [1948] P. 83 . $\quad$. $\quad$. 50

Argyll (Duchess) v. Argyll (Duke) [1967] Ch. $302 . \quad$. 143

Ash v. Ash [1972] Fam. $135 \quad$. $\quad . \quad 124$

Attorney-General v. Times Newspapers [1974] A.C. 273 . $\quad$. 69

Attwood v. Attwood [1968] P. $591 \quad 188$

In re B. (A Minor) (Adoption by parent) [1975] Fam. $127 \quad 236$

In re B. (An Infant) [1962] 1 W.L.R. 550; 1 All E.R. 872, C.A. .205, 208

In re B.(C.H.O.) (An Infant) [1971] 1 Q.B. 437 C.A.

234

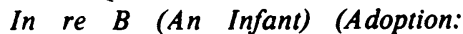
Parental Consent) [1976] Fam. $161 \quad . \quad . \quad .230$

In re B. (Infants) (Orse In re Baker
(Infants) [1962] Ch. 201

In re B. (M.F.) (An Infant) [1972] 1 W.L.R. $102 \quad$. . 237

In re B.(S.) [ 1968] Ch. $204 \quad$. .234, 235

In re B. (T.A.) (An Infant) [1971] Ch. 270

208 
B. v. Attorney-General (N.E.B. and others intervening) [1967] 1 W.L.R. 776; [ 1966] 2 All E.R. 145

B. v. B., The Times 29.11.72 . . 208

B. v. B., The Times 24.1.73 . . 205

B. v. B., The Times 15.5.75 C.A. .205, 207

B. v. B. (B. (An Infant) Intervening)

[1971] 1 W.L.R. 1486, 3 All E.R. 682, C.A.

207

B. (L.A.) v. B. (C.H.), The Times 18.2.75. $.145,216$

B. (M.) v. B. (R.) [1968] (Note) 1 W.L.R. 1182; 3 All E.R. 170 C.A.

B. v. B. (Practice Note) [1961] 1 W.L.R. 856; 2 All E.R. 296

In re Baker [1961] 1 Ch. 303 .

Baker v. Bolton (1808) 1 Camp. 493.

Baker v. Dalgleish S.S. Co. [1922] 1 K.B. 361 C.A.

In re Bailey, The Times, 12.10.76

Balfour v. Balfour [1919] 2 K.B. 57 C.A.

Banik v. Banik [1973] 1 W.L.R. 860; 3 All E.R. 45 C.A.

Bank of Montreal v. Stuart [1911] A.C. 120 (P.C.)

In re Barbour's Settlement Trusts [1974] 1 W.L.R. 1198; 1 All E.R. $1188 \quad$. $\quad$. $\quad$. $\quad 101$

Barnardo v. Ford [1892] A.C. $326 \quad .207,246$

Barnardo v. McHugh [1891] A.C. $388 \quad . \quad$. $\quad 207,224,226,246$

Barnes (R.M.) v. Barnes (G.W.) [1972] 1 W.L.R. 1381; 3 All E.R. 872 C.A.

Barritt v. Attorney-General [1971] 1 W.L.R. 1713; 3 All E.R. 1183 . 145, 220

Barron v. Willis [1899] 2 Ch. 578 . 25

In re Basioli [1953] Ch. 367 . . 148

Bassett v. Bassett [1975] Fam. 76 C.A. $.181,189$

Battle v. A.G. [1949] P. 358 . . 218

Baxter v. Baxter [1948] A.C. 274,

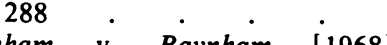

Baynham v. Baynham [1968] I W.L.R. 1890; [1969] 1 All E.R. 305 C.A.

Bazeley v. Forder (1868) L.R. 3 K.B. 559 C.A.

Beales v. Beales [1972] Fam. 210

Beamish v. Beamish (1861) 9 H.L. Cas. 274

Bedson v. Bedson [1965] 2 Q.B. 666 .

154
90

43,67

190
In re Beesley (A.) (A Bankrupt)

page [1975] 1 W.L.R. 568, 1 All E.R. 385

.90 Bell v. Bell [1961] 1 W.L.R. 1467 . 217 Bell v. Bell, The Times 10.12.75 .206, 208 Bendall v. McWhirter [1952] 2 Q.B. 466 C.A. . . . 93, 189, 190

Bennett v. Bennett (1879) 10 Ch.D. 474 . $\quad$.

Bennett v. Bennett [1952] 1 K.B. 249 C.A.

100

Bennett v. Bennett [1969] 1 W.L.R. 430; 1 All E.R. 539.

108

Benson v. Benson, The Times 2.1.73. Berthiaume v. Dastous [1930] A.C. 79

In re Besant (1879) 11 Ch.D. 508 . 72

Besant v. Wood (1879) 12 Ch.D.605 . 72

Best v. Samuel Fox [1952] A.C. 716 H.L.

66

Reg. v. Sec. of State for Home Dept. ex parte Bhajan Singh [1976] Q.B. 198

47

192

72
72

Reg. v. Bham [1966] 1 Q.B. 159

-Biggs v. Biggs and Wheatley [1976] 2 W.L.R. 942 .

Billington v. Billington [1974] Fam. 24 D.C.

$.123,144$

Bishop v. Cunard White Star Co., Ltd [1950] P. 240

155

Blackwell v. Blackwell, The Times 13.11.73 C.A.

132

Blake v. Midland Railway Co. (1852) 18 Q.B. 93

154

Blunt v. Blunt [1943] A.C. $517 \quad$. 113

In re Bluston [1967] Ch. $615 \quad . \quad .160,168$

Bomzee and Ledermann v. Bomzee

[1931] 1 Ch. 289 . . . 6, 25

Bothe v. Amos [1976] Fam. 46 C.A. .

Bowlas v. Bowlas [1965] P. 450

Bowman v. Bowman [1949] P. 352 C.A.

180

216

Reg. v. Bow Road Justices (Domestic Proceedings Court) ex parte Adedigba [1968] 2 Q.B. 572 C.A.

132

Boydell v. Gillespie (1970) 216 E.G. 1505.0.

Bradley v. Bradley [1973] 1 W.L.R. 1291, 3 All E.R. 750, C.A. . 70, 124

Brent v. Brent [1975] Fam. 1 . 71, 110, 181

Reg. v. Brentwood Supt. Registrar of Marriages, ex parte Arias [1968] 2 Q.B. 965 
Brett v. Brett [1969] 1 W.L.R. 1187; 1 All E.R. 1007, C.A.

Brickell v. Brickell [ 1974] Fam. 31 C.A.

Brister v. Brister [1970] 1 W.L.R. 664; 1 All E.R. 913.

Brockwell v. Brockwell, The Times 11.11 .75

Brooks v. Blount [1923] 1 K.B. 257 .

Brown v. Brown (1828) 1 Hag. Ecc. 523

Brown v. Brown, The Times 5.3.75 C.A.

Brown v. Draper [1944] 1 K.B. 309 C.A.

Browne (form. Pritchard) v. Pritchard [1975] 1 W.L.R. 1366 C.A.

Bryant v. Bryant, The Times 7.11.73 .

Buchanan v. Buchanan, The Times 21.5.73 C.A. .

Buckeridge v. Hall [1963] i Q.B. 613

Buckland v. Buckland [1968] P. 296.

Buckley v. John Allen and Ford (Oxford) Ltd [1967] 2 Q.B. 637

Bullock v. Bullock [1960] 1 W.L.R. 975; 2 All E.R. 307

Bullock v. Lloyds Bank Ltd [1955] Ch. 317 C.A.

Burgess v. Florence Nightingale Hospital for Gentlewomen [1955] 1 Q.B. 349

Burgess v. Rawnsley [1975] Ch. 429 C.A. .

Burke v. Burke [1974] 1 W.L.R. 1063; 2 All E.R. 944 C.A. $\quad .172,178$

Burns v. Edman [1970] 2 Q.B. 541 . Burvill v. Burvill, The Times 7.3.74

In re $C$. (A) (An Infant) [1970] 1 W.L.R. 288; 1 All E.R. 309 .208, 211

In re $C$. (L) [1965] 2 Q.B. 449 .

234

In re C. (M.A.) (An Infant) [1966] 1 W.L.R. $646 ; 1$ All E.R. 838 C.A.

C. v. C., The Times 28.5.71 . .

C. v. C., The Times 9.11 .72 . $\quad 205$

C. v. C. (Divorce: Interrogatories) [1973] 1 W.L.R. 568; 3 All E.R. 770

66,123

C. v. C. and C. (Legitimacy: Photographic Evidence) [1972] 1 W.L.R. 1335; 3 All E.R. 577 .
- Calder v. Calder, The Times 29.6.76

Calderbank v. Calderbank [1976] Fam. 93 C.A. .

In re Callaway [1956] Ch. 559

Caller v. Caller [1968] P. 37 D.C. . 216

Campbell v. Campbell [1976] Fam. 347

Caras v. Caras [1955] 1 W.L.R. 254; 1 All E.R. 624

174

184

Carr (M.) v. Carr (A.K.) [1974] 1 W.L.R. 1534; 1 All E.R. 1193 C.A.

Carew-Hunt v. Carew-Hunt, The Times 18.6.72

123

124

207

160

In re Catmull [1943] Ch. 262 Caunce v. Caunce [1969] 1 W.L.R. 286; 1 All E.R. 722

190

Chamberlain v. Chamberlain [1973] 1 W.L.R. 1557; [1974] 1 All E.R. 33 C.A.

180

Chaplin v. Brammall [1908] 1 K.B. 233

Chapman v. Chapman [1972] 1 W.L.R. 1544; 3 All E.R. 1089

Chard v. Chard [1956] P. 259 .

Chaterjee v. Chaterjee, [1976] Fam. 199 C.A.

Chaudhry v. Chaudhry (Note) [1976] 1 W.L.R. 221 C.A.

53,141

Cheni v. Cheni [1965] P. 85 . . 38, 40

Chipchase v. Chipchase [1939] P. 391 and [1942] P. 37

31

Clapham v. N.A.B. [1961] 2 Q.B. 77 .

Clarke v. Clarke [1943] 2 All E.R. 540

223

218

Cleary v. Cleary and Hutton [1974] 1 W.L.R. 73; 1 All E.R. 498 C.A.

In re Clore's Settlement Trusts [1966] 1 W.L.R. 955; 2 All E.R. 272 . Cobb v. Cobb [1955] 1 W.L.R. 731 C.A.

In re Cochrane (1843) 8 Dowl. 630

In re Cole [1964] Ch. 175 C.A.

Coleman v. Coleman [1973] Fam. 10

Colin Smith Music Co., Ltd v. Ridge [1975] 1 W.L.R. 463 C.A.

In re Collard's Will Trusts [1961] Ch. 293

Collett v. Collett $[1968]$ P $482^{\circ}$

In re Collins [1950] Ch. 498 C.A. 207

Collins v. Collins [1972] 1 W.L.R. 689; 2 All E.R. 658 C.A. .

16

4

23


Collister v. Collister [1972] 1 W.L.R. 54, 1 All E.R. 334 . .

Cooke v. Head [1972] 1 W.L.R. 518; 2 All E.R. 38 .

Cooke v. Head (No. 2) [1974] 1 W.L.R. 972; 2 All E.R. 1124

Cooper v. Crane [1891] P. 369

$.176,182$

Corbett v. Corbett (Orse Ashley) [1971] P. 83 .

Corfield v. Hodgson [1966] 1 W.L.R. 590; 2 All E.R. 205 .

Cowcher v. Cowcher [1972] 1 W.L.R. 425; 1 All E.R. 932 . . 95, 171, 179

Cowen v. Cowen [1946] P. 36 . . 67

Cowley (Earl of) v. Cowley (Countess of) [1901] A.C. 450 H.L. . .

Crabb v. Crabb (1834) 1 My \& K. 511

Cracknell v. Cracknell [1971] P. 356

Reg. v. Criminal Injuries Compensation Board [1972] 1 W.L.R. 569; 1 All E.R. 1034

In re Crippen [1911] P. 108

Crossland v. Hawkins [1961] Ch. 537

Reg. v. Croydon Juvenile Court Justices [1973] Q.B. 426 .

Cruse v. Chittum [1974] 2 All E.R.

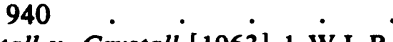

Crystall v. Crystall [1963] 1 W.L.R. 574; 2 All E.R. 330 C.A. .

48,139

Cumbers v. Cumbers [1974] 1 W.L.R. 1331; [1975] 1 All E.R. 1 C.A. . 128, 180

In re Cummins [1972] Ch. 62 C.A. . 99, 179

Curtis v. Curtis [1969] 1 W.L.R. 422; 2 All E.R. 207

Curwen v. James [1963] 1 W.L.R. 748; 2 All E.R. 619 C.A. .

Cutts v. Chumley [1967] 1 W.L.R. 742 C.A., [1968] 1 W.L.R. 668 (H.L.) .

Cuzner v. Underdown [1974] 1 W.L.R. 641 .

In re D. (A Minor) (Wardship: Sterilisation) [1976] Fam. 185

In re D. (An Infant) (Adoption: Parent's Consent) H.L. The Times 16.12.76

185

155

66

180

209

In re D. (An Infant) [1958] 1 W.L.R. 197, 1 All E.R. 427, C.A.

In re D. (Infants) [1970] 1 W.L.R. 599; 1 All E.R. 1088 C.A.

In re $D$. (Minors) (Adoption by parent) [1973] Fam. 209.
In re D. (Minors) (Wardship: Jurisdiction) [1973] Fam. 179 .

page

In re D. (S.L.) (An Infant) [1972] 1 W.L.R. 102; 1 All E.R. 898

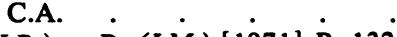
D. (J.R.) v. D. (J.M.) [1971] P. 132 'D. v. N.S.P.C.C. [1976] 3 W.L.R. 124 C.A.

Reg. v. $D$. (a boy) The Times 21.10.76, C.A.

Dalrymple v. Dalrymple (1811) 2 Hagg. Con. 54

210

237

215

209

262

50

Dalhousie v. M'Dovall (1840) $7 \mathrm{Cl}$ \& F. 817 .

217

Dancer v. Dancer [1949] P. $14 \dot{2} \quad$. 31

Danchevsky v. Danchevsky [1975] Fam. 17 C.A. . . . . $\quad .171,192$

Darvill v. Darvill, The Times 15.2.73 . 127

Daubney v. Daubney [1976] Fam. 267

178

Davies v. Powell Duffryn Associated Colliers Ltd [1942] A.C. 601 .

Davis v. Vale [1971] 1 W.L.R. 1022; 2 All E.R. 1021 C.A.

Reg. v. Davies (Peter) [1975] Q.B. 691

de: Please see substantive name

Reg. v. Deacon [1973] 1 W.L.R. 696; 2 All E.R. 1145 C.A.

155

95

70

Debenham v. Mellon (1880) 6 Ap. Cas. 24 H.L.

R. v. Delavel (1763) 2 Burr. 1434

In re Dellow's Will Trusts [1964]

1 W.L.R. 451; 1 All E.R. 771

Des Salles d'Epinoix v. Des Salles d'Epinoix [1967] 1 W.L.R. 553; 2 All E.R. 539 C.A.

136

Des Salles d'Epinoix v. Kensington and Chelsea R.B.C. [1970] 1 W.L.R. 179; 1 All E.R. 18

Diwell v. Farnes [1959] 1 W.L.R. 624; 2 All E.R. 379 C.A. .

91

211

151

Doherty v. Doherty [1976] Fam. 71 C.A.

Dorrell v. Dörell [1972] 1 W.L.R. 1087; 3 All E.R. 343

Dowell v. Dowell [1952] 2 All E.R. 141 D.C.

Downing v. Downing [1976] Fam. 288 . . . .

Dredge v. Dredge [1947] 1 All E.R. 29

Dryden v. Dryden [1973] Fam. 217 .

Dyson Holdings v. Fox [1976] Q.B. 503
182

182

184

129

185

218

133

152 
In re E. (An Infant) [1956] Ch. 23

In re E., The Times 24.3.60

In re E.O., The Times 16.2.73 .

Earley v. Earley, The Times 20.6.75 C.A.

In re Eichholz [1959] Ch 708

Evans v. Evans 10.7.76, Bar Library Transcript No. 56

Eves v. Eves, [1975] 1.W.L.R. 1338, 3 All E.R. 768, C.A.

In re $F$. (T.) (An Infant) [1970] 1 W.L.R. 192; 1 All E.R. 344

In re $F$ (A Minor) (Wardship: Appeal) [1976] Fam. 238 C.A.

-In re F (orse. A.) (A Minor) (Publication of Information) [1976] 3 W.L.R. 813 C.A. .

F. v. B., The Times 19.10 .76

F. v. F. [1971] P. 1

Falconer v. Falconer [1970] 1 W.L.R. 1333; 3 All E.R. 449 C.A.

In re Ferrar's Application [1966] Ch. 126 C.A.

In re Figgis [1969] 1 Ch. 123 . .

Fowler v. Fowler \& Sine [1964] P. 311 C.A.

Franklin v. S.E. Railway (1858) 3 H. \& N. 211

French v. Gething [1922] 1 K.B. 236 C.A.

Fribance v. Fribance (No. 2) [1957] 1 W.L.R. 384 C.A.

Fuller v. Fuller [1973] 1 W.L.R. 730; 2 All E.R. 650 C.A.

In re G. (Infants) [1963] 1 W.L.R. 1169; 3 All E.R. 370

G. v. G. (1871) 2 P. \& D. 287

G. v. G. [1924] A.C. 349

Gaines v. W. [1968] 1 Q.B. 782

In re Geering [1964] Ch. 136 .

Gengler v. Gengler [1976] I W.L.R.

$112,173,188$

In re Giles [1972] Ch. 544

Giltrow v. Day [1965] 1 W.L.R. 317 . 221

Gissing v. Gissing [1971] A.C. 886 . 94, 95,

Glenn v. Glenn [1973] 1 W.L.R. 1016; 2 All E.R. 1187 . . 25, 172

In re Goodman's Trusts (1881) 17 Ch.D. 266 C.A.

Goodburn v. Thomas Cotton Ltd [1968] 1 Q.B. 845 C.A. : Goodrich v. Goodrich [1971]
1 W.L.R. 1142; 2 All E.R. 1340

In re Goodwin [1969] 1 Ch. 283
45,210

221

134

94

160

88-9

179

127

210

43

43

221
155,156

123 161
Gordon v. Gordon, The Times 12.10.73 C.A. $\quad$. . . 25, 172

Gorulnik v. Gorulnik [1958] P. 47 C.A.

189

Grand Trunk Railway Co. of Canada v. Jennings (1888) 13 App. Cas.

Gray v. Cathcart (1922) 38 T.L.R. 562 . $324^{\circ}$

- Gray v Gray [1976] Fam. $32 \dot{1} 92$ 153,184

Green v. Russell \1959] 2 Q.B. 226 . 154

R. v. Greenhill (1836) 4 Ad. \& E. 624

In re Gregory deceased [1970] 1 W.L.R. 1455 C.A.

13,55

Grein v. Imperial Airways Ltd [1937] 1 K.B. 50 C.A.

160,161

Griffiths v. Griffiths [1974] 1 W.L.R. 1350; 1 All E.R. 932 C.A. .178, 185

Grigson v. Grigson, The Times 6.11.73 C.A. . . . . 128

Gurasz v. Gurasz [1970] P. 11. C.A. .136, 189 In re Gyngall [1893] 2 K.B. 232 C.A.

246

In re H. (G.J.) (An Infant) [1966] 1 W.L.R. 706; 1 All E.R. 952

H. v. H. [1954] P. 258

H. v. H., The Times 13.11.76 . . 206

H. v. H. (Child: Judicial Interview) [1974] 1 W.L.R. 595; 1 All E.R. 1145 C.A.

204

H. v. H. (Family Provision: Remarriage) [1975] Fam. 9

178

H. v. H., The Times 7.7.75 C.A. . 208

Hakluytt v. Hakluytt [1968] 1 W.L.R. 1145; 2 All E.R. 868 C.A.

185

Hale v. Hale [1975] 1 W.L.R. 931 C.A.

71,181

Halifax Building Society v. Clark [1973] Ch. 307

190

Hall v. Hall (1908) 24 T.L.R. $756 \quad$. 46

Hall v. Hall [ 1963] P. 378 C.A. $\quad 210$

Hall v. Hall [1971] 1 W.L.R. 404; 1 All E.R. 762 C.A. $\quad . \quad 181,189$

In re Hamer's Estate [1937] 1 All E.R. 130

219

In re Hardy's Trust, The Times 23.10.70

172

Harnett v. Harnett [1973] Fam. 156, and [1974] 1 W.L.R. 219 C.A. 173,

Harrods v. Stanton [1923] 1 K.B. $174,175,180$ 516 D.C.

88

Harrods v. Tester [1937] 2 Ail E.R. 236 C.A. 
Harthan v. Harthan [1949] P. 115 C.A.

Har-Shefi v. Har-Shefi (No. 2) [1953] P. 220 .

Harvell v. Foster [1954] 2 Q.B. 367 C.A.

Hashmi v. Hashmi [1972] Fam. 36 .

Hastings \& Thanet Building Society v. Goddard [1970] 1 W.L.R. 1544; 2 All E.R. 737, C.A. . 190, 191

Hawes v. Evendon [1953] 1 W.L.R. 1169, 2 All E.R. 737 C.A.

Hawkins v. A.-G. [1966] 1 W.L.R. 978; 1 All E.R. 392.

Hay v. Hughes [1975] Q.B. 790 C.A.

Hayward v. Hayward [1961] P. 152 .

Heatley v. Steel Co. of Wales [1953] 1 W.L.R. 405; 1 All E.R. 489 C.A.

Hector v. Hector [1973] 1 W.L.R. $1122 ; 3$ All E.R. 1070 C.A.

Henderson v. Henderson and Crellin [1944] A.C. 49

Hickson v. Hickson [1953] i Q.B. 420.

Hirschhorn v. Evans [1938] 2 K.B. 801

Hislop v. Hislop [1950] W.N. 124 .

Hoddinott v. Hoddinott [1949] 2 K.B. 406 C.A.

In re Hodgkinson [1967] Ch. 634 C.A.

Holdanowski v. Holdanowska [1957] P. 301 C.A.

Holmes v. Holmes [1966] 1 W.L.R. 187; 1 All E.R. 356 .

Hooper (Orse Harrison) v. Hooper [1959] 1 W.L.R. 1021; 2 All E.R. 575

Hopes v. Hopes [1949] P. 227.

Horrocks v. Forray [1976] 1 W.L.R. 230; 1 All E.R. 737 C.A. . .

Horton v. Horton (1948) 64 T.L.R. 62, [ 1947] 2 All E.R. 871 H.L. .

Hounslow London Borough Council v. Peake [1974] 1 W.L.R. 26; 1 All E.R. 688

Howes v. Bishop [1909] 2 K.B. 390 C.A.

Hughes v. Griffin [1969] 1 W.L.R. 23; 1 All E.R. 460 C.A.

Humphrey v. Ward Engineering Services, The Times 28.5.75

Humphreys v. Polak [1901] 2 K.B. 385 page
Hunter v. Hunter [1973] 1 W.L.R. 958; 3 All E.R. 362 C.A. $\quad .173,185$ In re Hurd [1941] Ch. 196 . . 148 In re Hurll [1952] Ch. 722 . . 218

Hussey v. Palmer [1972] 1 W.L.R. 1286; 3 All E.R. 744 C.A. . Huxford v. Huxford [1972] 1 W.L.R. 210; 1 All E.R. 330 .

182

Hyde v. Hyde and Woodmansee (1866) L.R. 1 P. \& M. 30 .

Hymns v. Hymns, (Practice Note) [1971] 1 W.L.R. 1474; 3 All E.R. 596

Iddenden (Orse Brains) v. Iddenden [1958] 1 W.L.R. 1041, 3 All E.R. 241

Imam Din v. N.A.B. [1967] 2 Q.B. 213

Reg. v. Chief Immigration Officer, ex parte Salamat Bibi [1976] 1 W.L.R. 979 C.A. .

In re J. (Adoption Order: Conditions) [1973] Fam. $106 \cdot \cdot 237$

J. v. C. [1970] A.C. 668 . $\quad$. 19, 206, 207

J. v. J. [1947] P. $158 \quad$. $\quad$. $\quad$. 67

Reg. v. Jackson (The Clitheroe Case) [1891] 1 Q.B. 671 C.A. . 55, 60, 70 Jackson v. Jackson [1924] P. $19 \quad$. 127

Jackson v. Jackson [1971] 1 W.L.R. 1539; 3 All E.R. 774, C.A.

Jackson v. Jackson [1973] Fam. 99 . 182

Jansen v. Jansen [1965] P. 478 : 95

Jeffrey v. Johnson [1952] 2 Q.B. 8 C.A.

222

Jodla v. Jodla [1960] 1 W.L.R. 236; 1 All E.R. 625

44,61

In re Johns' Assignment Trusts [1970] 1 W.L.R. 955; 2 All E.R. 210

Jones v. Challenger [1961] i Q.B. 176 C.A.

91,172

Jones v. Evans [1944] 1 K.B. 582 . 221

Jones v. Jones [1971] 1 W.L.R. 396; 2 All E.R. 737 C.A.

Jones v. Jones [1971] 3 All E.R. 1201 C.A.

189

Jones (D.I.) v. Jones (E.T.) [1972] 1 W.L.R. 1269; 3 All E.R. 289 .

Jones (E.G.) v. Jones (E.F.) [1974] 1 W.L.R. 1471; 3 All E.R. 702, C.A.

Jones (M.A.) v. Jones (W.) [1976] Fam. 8 C.A.

Jones v. Maynard [1951] Ch. 572

Julian v. Julian, The Times 4.10.72 . 129

Jussa v. Jussa [1972] 1 W.L.R. 881; 2 All E.R. 600
186

182

206

180

208 
In re K. [1965] A.C. 201

In re K. [1966] 1 W.L.R. 1241; 3 All E.R. 154

K. v. J.M.P. Co. Ltd [1976] Q.B. 85

Kassim v. Kassim [1962] P. 224 .

Katz v. Katz [1972] 1 W.L.R. 955; 3 All E.R. 219

Kaur v. Singh [1972] 1 W.L.R. 105; 1 All E.R. 292 C.A.

In re Kay [1965] 1 W.L.R. 1463

Kelly v. Kelly (1933) 49 T.L.R. 99

Kelner v. Kelner [1939] P. 411

Kenward v. Kenward [1951] P. 124 C.A.

Kirke v. Kirke [1961] 1 W.L.R. 1411; 3 All E.R. 1059

Knowles v. Knowles [1962] P. 161

Koppel v. Koppel, Wide, Claimant [1966] 1 W.L.R. 802; 2 All E.R. 187 C.A.

Kochanski v. Kochanska [1958] P. 147

Kowalczuk v. Kowalczuk [1973] 1 W.L.R. 930; 2 All E.R. 1042

C.A. . . . . $95,171,179$

Kremezi v. Ridgeway [1949] I All E.R. 662

Krishnan v. Sutton L.B.C. [1970] Ch. 181, C.A.

Krystman v. Krystman [1973] 1 W.L.R. 927, 3 All E.R. 247 C.A.

In re L. (An Infant) (Practice Note) [1963] 1 W.L.R. 97; 1 All E.R. 176

In re L. (Infants) [1962] 1 W.L.R. 886; 3 All E.R. 1 C.A. .

In re L. (Minors) (Wardship: Jurisdiction) [1974] 1 W.L.R. 250; 1 All E.R. 913 C.A. .

L. v. L. (1922) 38 T.L.R. 697 .

L. v. L. [1949] 1 All E.R. 14

R. v. Lamb (1934) 50 T.L.R. 310

Lang v. Lang [1955] A.C. 402 P.C.

Lanitis v. Lanitis [1970] 1 W.L.R. 503; 1 All E.R. 466 .

Law. v. Gustin, [1976] Fam 155

Lazarewicz v. Lazarewicz [1962] P. 171

Leake v. Bruzzi [1974] I W.L.R. 1528; 2 All E.R. 1196 C.A.

Reg. v. Leggatt (1852) 18 Q.B. 781

- Leicestershire County Council v. Cross [1976] 2 All E.R. 491

Lincoln Corpn. v. Parker [1974] 1 W.L.R. 713; 2 All E.R. 949 .

209

210

156

46

124

44

160,168

46

6

46

77

217

89

51

260 page Parental Consent) [1973] Q.B. 108 C.A.

M. v. M., The Times 30.11 .72 .

M. v. M. (Child: Access) [1973] 2 All E.R. 81 D.C.

In re McCarthy [1975] 1 W.L.R. 807; 2 All E.R. 857

page Justice ex parte M. (A Minor), [1976] Q.B. 957

259

e Ann Lloyd (1841) 3 M. \& G. 547

Lloyds Bank v. Savory [1933] A.C.

58

IW.L. 1276; 3 All ER. 625

173 ,

176,184

Lord Advocate v. Jaffrey [1921] A.C. 146

v. Lowe [1973] Q.B. 702 C.A. . 247

Lowry v. Lowry [1952] P. 252 . . 108, 115

In re $M$. (An Infant) [1955] 2 Q.B. 479 C.A.

$.224,231$

In re $M$. (An Infant) [1961] Ch. 328 C.A.

210

207

234

205

207

MacDarmaid v. MacDarmaid [1950] P. 218

90,171

In re McGeorge [ 1963] Ch. $544 \quad 97$

McGibbon v. McGibbon [1973] Fam. 170 . . . . 71, 133, 136 McGill v. Robson [1972] 1 W.L.R. 237; 1 All E.R. 362.

127

In re $M c K .$, The Times, 14.7.76

195

Mackenzie v. Royal Bank of Canada [1934] A.C. 468 P.C.

25

McLeod v. McLeod, The Times 3.7.73

Madden v. Madden [1974] 1 W.L.R. 247; 1 All E.R. 673.

71

182

Malyon v. Plummer [1964] 1 Q.B. 330

154

R. v. de Manneville (1804) 5 East 221

$13,55,211$

Mapp. v. Oram [1970] A.C. $362 \quad$. 82

Marsden (J.L.) v. Marsden (A.M.) [1973] 1 W.L.R. 641; 2 All E.R. 851

177

Marshall v. Crutwell (1875) L.R. 20 Eq. 328 .

Martin v. Martin [1976] Fam.

94 335 C.A.

178,185 
Mason v. Mason [1972] Fam. 302

-Matcham v. Matcham, The Times 30.6.76 .

Mathias v. Mathias [1972] Fam. 287 C.A.

Maynard v. Maynard [1969] P. 88

Mehta v. Mehta [1945] 2 All E.R. 690

Merker v. Merker [1963] P. 283 .

Merritt v. Merritt [1970] 1 W.L.R. 1211; 2 All E.R. 760 C.A.

Mesher v. Mesher, The Times 13.2.73 C.A.

Metrobarn Litd v. Gehring [1976] 1 W.L.R. 776 C.A. .

$\cdot .189,191$

Middleton v. Baldock [1950] I K.B. 657

Miles v. Bull [1969] 1 Q.B. 258

Miller v. Miller [1961] P. 1

Reg. v. Miller [1954] 2 Q.B. 282

Milliken-Smith v. Milliken-Smith [1970] 1 W.L.R. 973; 2 All E.R. 560 C.A.

Reg. v. Millis (1844) $10 \mathrm{Cl}$. \& Fin. 534

Millward v. Millward [1971] 1 W.L.R. 1432; 3 All E.R. 526 C.A

Millward v. Shenton [1972] 1 W.L.R. 711 .

- Ex parte Minhas, Reg. v. Registrar General of Births, etc. [1976] 2 W.L.R. 473 .

In re Mohamed Arif [1968] Ch. 643 C.A.

Montgomery v. Montgomery [1965]

P. $46 . \quad . \quad 70,136,189$

Morgan v. Morgan, The Times 24.2.73 .

Morley v. Morley [1961] 1 W.L.R. 211; 1 All E.R. 428.

Morris v. Tarrant [1971] 2 Q.B. 143.

Morss v. Morss [1972] Fam. 264 C.A.

Mouncer v. Mouncer[1972] 1 W.L.R. 321; 1 All E.R. 289.

Mourton v. Hounslow London Borough Council [1970] 2 Q.B. 362 D.C.

Murphy v. Ingram [1974] Ch. 363 C.A.

Mustafa v. Mustafa [1975] I W.L.R. 1277

In re N. (Infants) [1967] Ch. 512

In re N. (Minors), The Times 13.6.73. page

93,188

190

113

70

175

$8,29,51$

185

161

141

210

126

108

189

186

127

191

82

124

209

198
In re N. (Minors) (Parental Rights) [1974] Fam. 40

page N. v. N. [1964] 108 S.J. $99 \quad$. $\quad$. 134 Nanda v. Nanda [1968] P. 35 . $\quad$. 116 Napier v. Napier [1915] P. 184, 192-3 Nash v. Nash [1973] 2 All E.R. 704 C.A.

216

Reg. v. Nash (1883) 10 Q.B.D. 454 C.A. . . . . 72, 224, 226

Nast v. Nast [1972] Fam. 142 C.A. . 66, 123

National Assistance Board v. Mitchell [1956] 1 Q.B. 53 D.C.

National Assistance Board v. Parkes [1955] 2 Q.B. 506 C.A.

National Assistance Board v. Prisk [1954] 1 W.L.R. 443; 1 All E.R. 400

National Assistance Board v. Tugby [1957] 1 Q.B. 506

223

National Assistance Board v. Wilkinson [1952] 2 Q.B. 648

National Provincial Bank v. Ainsworth [1965] A.C. 1175

93, 94, 178,189

Newbould v. A.-G. [1931] P. $75 \quad$. 218

Newgrosh v. Newgrosh [1950] 100 L. Jo. 525 C.A. . . 95

Newman v. Newman [1971] P. $43 \quad .199,216$

In re Nicholson deceased [1974]

1 W.L.R. 476; 2 All E.R. $386 \quad .173,179$

Nielsen-Jones v. Fedden [1975] Ch. 222

172

Reg. v. Noble [1974] 1 W.L.R. 894; 2 All E.R. 811 C.A.

Reg. v. Notts. Justices ex parte Bostock [1970] I W.L.R. 1117; 2 All E.R. 641

222

In re $O$. (An Infant) The Times 17.2.63 C.A. .

In re O. (Infants) [1962] 1 W.L.R. 724 C.A.

$.208,211$

In re O. (Infants) [1971] Ch. $748^{\circ}$ C.A.

O'Brien v. O'Brien and Smith [1972] Fam. 20

205

$O$ 'Connor v. $A$ and B. [1971] 1 W.L.R. 1227 H.L. (Sc.) [1971]

$O^{\prime} D . \quad$ v. $O^{\prime} D . \quad[1976]$ Fam. 83 C.A.

187

Old Gate Estates v. Alexander [1950] 1 K.B. 311 C.A.

175

Oldfield v. National Assistance Board [1960] 1 Q.B. 635

93,188

O'Neill v. O'Neill [1975] 1 W.L.R. 1118; 3 All E.R. 289 C.A. 
In re P. (A.J.) (An Infant) [1968] 1 W.L.R. 1976

In re P. (Infants) [1962] 1 W.L.R. 1296

In re P. (Infants) [1967] 1 W.L.R. 818; 2 All E.R. 229.

P. v. L. (1873) 3 P. \& D. 73n.

P. v. Mck, The Times 28.6 .73 .

P. v. P. [1969] 1 W.L.R. $898 ; 3$ All E.R. 777

P. v. P. and J. [1971] P. 217 C.A.

P. (L.E.) v. P. (J.M.) [1971] P. 318

-Pace (formerly Doe) v. Doe [1976]

$$
3 \text { W.L.R. } 865 \text {. }
$$

Parghi v. Parghi, The Times 8.5.73

In re Park [1954] P. 112 C.A. .

Parker v. Parker [1972] Fam. 116.

Parkes v. Parkes [1971] 1 W.L.R. 1481; 3 All E.R. 870 C.A.

In re Parkinson, The Times 4.10.75 .

Parojcic (Orse Ivetic) v. Parojcic [1958] 1 W.L.R. 1280; [1959] 1 All E.R. 1

Parsons v. Parsons [1975] 1 W.L.R. 1272

In re Pauling's Settlement Trusts (No. 2) [1963] Ch. 576

In re Pauling's Settlement Trusts [1964] Ch. 303 C.A.

Payne v. Critchley and the National Assistance Board [1962] 2 Q.B. 83 .

Payne-Collins v. Taylor Woodrow Constructions Ltd [1975] Q.B. 300

In re Peacock [1957] 1 Ch. 310

In re Peete [1952] 2 All E.R. 599

Penhas v. Tan Soo Eng [1953] A.C. 304 P.C.

Penn v. Dunn [1970] 2 Q.B. $686^{\circ}$ C.A.

Perry v. Dembowski [1951] 2 K.B. 420 C.A.

Pettitt v. Pettitt [1963] P. 177 C.A. .

Pettitt v. Pettitt [1970] A.C. $777.90,94,177,179$

Pevec v. Brown (1964) 108 S.J. 219 .

Pheasant v. Pheasant [1972] Fam. $202 . \quad . \quad$.

Phillips v. Phillips [1973] 1 W.L.R. 615; 2 All E.R. 432 C.A. .

In re Pilkington [1964] A.C. 612

In re Plomley (1882) 47 L.T. (N.S.) 283

Plummer v. Plummer [1917] P. 163 .

Poel v. Poel [1970] 1 W.L.R. 1469; 3 All E.R. 659 C.A.
In re Pointer [ 1941] Ch. 60

In re Pollock [1941] Ch. 21

Poole v. Stokes (1914) W.N. 123

Porter v. Porter [1969] 1 W.L.R. 1155; 3 All E.R. 640 C.A.

page

160

151

246

135

\section{PRACTICE DIRECTIONS}

- (Decrees and Orders: Agreed terms) [1972] 1 W.L.R. 1313; 3 All E.R. 704 .

- (Deed Poll) (Minors) [1969] 1 W.L.R. 1330; 3 All E.R. 288 .

- (Divorce: Children) [1971] 1 W.L.R. 10; [1970] 3 All E.R. 1023

- (Divorce: Proof of Adultery) (No. 2) [1973] 1 W.L.R. 1052; 3 All E.R. 180

- (Divorce: Reconciliation) (No. 2) [1972] 1 W.L.R. 1309; 3 All E.R. 768

- (Divorce: Registry: Consent Summons) [1974] 1 W.L.R. 937; 2 All E.R. 1120

- (Matrimonial Causes: Injunction) [1974] 1 W.L.R. 936; 2 All E.R. 1119

- (Matrimonial Causes: R.C.J.) [1973] 1 W.L.R. 554; 2 All E.R. 288

- Matrimonial Causes: Special Procedure) [1973] 1 W.L.R. 1442; 3 All E.R. 1182

- Matrimonial Causes: Special Procedure Extension) [1975] 1 W.L.R. 1594

- (Petition for Declaration) [1971] 1 W.L.R. 29; 3 All E.R. $1024 \quad .117,136$

- (Petition) (Petitioner's Address) [1975] 1 W.L.R. 787; 2 All E.R. 384

- Variation of Trusts: Counsel's Opinion [1976] 1 W.L.R. 884

- (Wardship Applications) [1966] 1 W.L.R. 1384; 3 All E.R. 144

- (Wardship Applications) [1967] 1 W.L.R. 623; 1 All E.R. 828

186

PRACTICE NOTES

- (Disclosure of Addresses) [1973] 1 W.L.R. 60

- (Divorce: Conciliation) [1971] 1 W.L.R. 223 
PRACTICE NOTES contd.

- (Infants: Matrimonial Causes) (No. 2) [1972] 1 W.L.R. 1195; 3 All E.R. 255 .

-(Inheritance: Family Provision) [1976] 1 W.L.R. 418

-(Matrimonial Causes: Issues) [1975] 1 W.L.R. $1640 \quad . \quad .144,216$

In re Preston deceased [1969] 1 W.L.R. 317; 2 All E.R. 961

Preston v. Preston [1963] P. 411 C.A.

Prior v. Prior (1970) 114 Sol. Jo. 72 .

In re Pugh [1943] Ch. 387

Pulford v. Pulford [1923] P. 18

Qureshi v. Qureshi [1972] Fam. 173.

In re R. (Adoption) [1967] 1 W.L.R. 34; [ 1966] 3 All E.R. 613

In re R. (An Infant) [1974] 1 All E.R. 1033, The Times 18.1.74.

In re $R$. (K.) [ 1964] Ch. 455 . .

In re R. (M.J.) (A Minor) (Publication of Transcript) [1975] Fam. 89

In re R. (P.M.) (An Infant) [1968] 1 W.L.R. 385, 1 All E.R. 691

R. v. R. (Blood test: Jurisdiction) (Practice Note) [1973] 1 W.L.R. 115; 3 All E.R. 933.

Race Relations Board v. Applin [1975] A.C. 259

Radwan v. Radwan (No. 2) [1973] Fam. 85

Ramsey v. Margrett [1894] 2 Q.B. 18

Ramsay-Fairfax v. Ramsay-Fairfax [1956] P. 115

Rawlings v. Rawlings [1964] P. $398 \quad .172,178$

Razelos v. Razelos (No. 2) [1970] 1 W.L.R. 392; 1 All E.R. 386

In re: Please see substantive name

Regan v. Williamson [1976] 1 W.L.R. 305 .

154,156

Reg. v.; R. v. (Please see defendant's name)

-Reg. v. Registrar General of Births, etc. ex parte Minhas, [1976] 2 W.L.R. 473 .

Reg v. Registrar General ex parte Segerdal [1970] 1 Q.B. 430

Reg. v. Reid [1973] Q.B. 229 C.A.

-Reiterbund v. Reiterbund [1975] Fam. 99 C.A. .

de Reneville v. de Reneville [1948] P. 100 , C.A.
Restall v. Restall (1929) 45 T.L.R. 518 .

Richards v. Dove [1974] 1 All E.R. 888

Richards v. Richards [1972] 1 W.L.R. 1073; 3 All E.R. 695 .

Rimmer v. Rimmer [1953] 1 Q.B. 63 C.A.

Robinson v. Williams [1965] i Q.B.

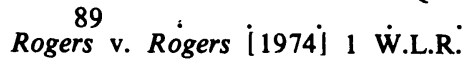
709; 2 All E.R. 361 C.A. .

page

40

182

124

95

222

124

Rondeau, Le Grand v. Marks [1918] 1 K.B. 75 C.A.

Roper v. Roper [1972] 1 W.L.R. 1314. .

Ross v. Pearson [1976] 1 W.L.R. $224 . \quad . \quad . \quad . \quad .186,188$

Rukat v. Rukat [1975] Fam. 63 C.A.

130

Rule v. Rule [1972] 1 W.L.R. 218; 3 All E.R. 1368

128

Rutherford v. Rutherford [1970] 1 W.L.R. 1479; 3 All E.R. 422 .

Ryan v. Ryan, The Times 4.5.63 $\quad 182$

In re S. [1973] 3 All E.R. 88 . . 234

In re S. The Times 17.11 .76 C.A. $\quad 230$

In re S. (A Minor) (Adoption Order: Access) [1976] Fam. 1 C.A.

In re $S$. (An Infant) [1965] 1 W.L.R. 483; 1 All E.R. 865 C.A. .

-In re $S$. and A., F. (Infants) The Times 21.12.76, C.A.

89

237

210

236

In re S. (Infants) [1967] 1 W.L.R. 396; 1 All E.R. 202 . . 204, 209, 219

S. v. F. [1967] 1 Q.B. $367 \quad$. 221

S. v. Huddersfield Borough Council [1975] Fam. 113 C.A.

S. v. McC. (Orse S.) and M. (D.S. Intervener) $W$. v. $W$. [1972] A.C. 24

237

S. v. S. [1962] 1 W.L.R. $445 ; 2$ All E.R. 1, C.A. .

"S. v. S., The Times 23.6.73

S. v. $S$ [1976] 3 W L R 775, C A 173,185

S. v. S. (Note) [1976] Fam. 18. . 77, 180

S. v. S. (Orse C.) [1956] P. 11 . . 43

$S$. v. S. and $W$. v. Official Solicitor [1972] A.C. 24

S.Y. v. S.Y. (Orse W.) [1963] P. 37 C.A. and [1962] 1 W.L.R. 1012 (H.L.)

-Reg. v. Sagoo [1975] 3 W.L.R. 267 C.A.

Reg. v. Chief Immigration Officer, ex parte Salamat Bibi [1976] 1 W.L.R. 979 C.A. .
216

207

92

43

41,53

62 
Table of Cases $\quad \mathbf{x x x i i i}$

Salveson v. Administrator of Austrian Property [1927] A.C. 641

Samson v. Samson [1960] 1 W.L.R. 190; 1 All E.R. 653 C.A.

In re Sanderson deceased, The Times 2.11.63.

Santos v. Santos [1972] Fam. 247 C.A.

Schlesinger v. Schlesinger [1960] P. 191

Scott v. Sebright (1886) 12 P.D. 21.

Scrimshire v. Scrimshire (1752) 2 Hagg. Con. 395

Reg. v. Secretary of State for Home Dept. ex parte Phansopkar [1976] Q.B. 606 C.A.

Shahnaz v. Rizwan [1965] 1 Q.B. 390

In re Shanahan [1973] Fam. 1

Shaw v. Shaw [1954] 2 Q.B. 429 C.A.

Shepherd v. Cartwright [1955] A.C. 431 H.L.

Shepherd v. Pullbrook (1888) 4 T.L.R. 642

Sheward v. A.-G. [1966] 1 W.L.R. 978

In re Sigsworth [1935] Ch. 89 .

Silver v. Silver [1955] 1 W.L.R. 728 .

Silverstone v. Silverstone [1953] P. 174

Sim v. Sim [1944] P. 87 .

Simpson v. Collinson [1964] 2 Q.B. 80 C.A.

Singh v. Singh [ 1971] P. 226 C.A.

Smallman v. Smallman [1972] Fam. 25 C.A. .

Smith v. Smith, The Times 15.12.73.

Snow v. Snow [1972] Fam. 74.

In re Solomon (A Bankrupt) [1967] 1 Ch. 573

In re Somech [1957] Ch. 165 . .

Somerset County Council v. Brice [1973] 1 W.L.R. 1169; 3 All E.R. 438

Somerset County Council v. Kingscott [1975] 1 W.L.R. 283; 1 All E.R. 326

Reg. v. Southwark Juvenile Court [1973] 1 W.L.R. 1300; 3 All E.R. 383

Sowa v. Sowa [1961] P. 70 C.A.

Spellman v. Spellman [1961] 1 W.L.R. 921; 2 All E.R. 498; C.A.

Re Spence (1847) 2 Phil. 247 page

100

45,61

189

140

222

46

170-1

125

216

90,171

98

260

260

260

52
Spiro v. Lintern [1973] 1 W.L.R. 1002; 3 All E.R. 319 C.A.

page

Stewart v. Stewart [1974] 1 W.L.R. 877; 2 All E.R. 795 C.A. .

Stewart v. Stewart [1973] Fam. 21 . 136,

189,217

Stocker v. Stocker [1966] 1 W.L.R. 190; 2 All E.R. 147 . . . 219

Springfellow v. Springfellow [1976] l W.L.R. 645 C.A. . . . 126

In re Styler [1942] Ch. $388 \quad . \quad .160,161$

Surrey County Council v. S. [1974] Q.B. 124

258

Sykes v. N.E. Railway (1875) 44 L.J.C.P. 191 .

154

$\begin{array}{lr}\text { In re Sylvester [1941] Ch. } 87 \text {. } & 160 \\ \text { Szechter v Szechter [1971] P. 286 } & 45,61\end{array}$

$\begin{array}{lrr}\text { Szechter v. Szechter [1971] P. } 286 & . & 45,61 \\ \text { In re T., The Times 16.1.74 } & . & 204\end{array}$

In re T. (A.J.J.) [1970] Ch. 688 C.A. . . . 210, 253

In re T. (Orse H.) (An Infant) [1963] Ch. 238 . $\quad . \quad$. $57,58,199$

T. (H.H.) v. T. (E.) [1971] 1 W.L.R. 429; 1 All E.R. 590 .

219

T. v. T., The Times 4.11 .70 C.A. $\quad$. 216

T. v. T., The Times 4.11.74 · . . 183

Taczanowska v. Taczanowski [1957] P. 301 C.A.

51

Talbot v. Talbot, (1967) 111 Sol. Jo. 213

42

Talbot v. Talbot, The Times 19.10.71 . 129

Tanner v. Tanner [1975] 1 W.L.R. 1346 C.A. $\quad . \quad$. $178,182,221$

Tarr v. Tarr [1973] A.C. 254 . $\quad$. 191

In re Taylor [1961] 1 W.L.R. 9; 1 All E.R. 55 C.A. .

30

In re Taylor's Application [1972] 2 Q.B. 369 C.A.

69

Taylor v. O'Connor [1971] A.C. 115 .

Temple v. Temple [1976] 1 W.L.R. 701 C.A.

155

In re Thain [1926] Ch. 676 C.A. $\quad$. 204

Thomas v. Thomas, The Times 13.12.72 C.A.

123

Thompson v. Earthy [1951] 2 K.B. 598

Thompson v. Price [1973] Q.B. 838 .

Thompson v. Thompson, The Times 12.3.75 C.A.

Thompson v. Thompson [1976] Fam. 25 C.A. .

In re Thornley deceased [1969] 1 W.L.R. 1037; 3 All E.R. 31, C.A.

Thurlow v. Thurlow [1976] Fam. 32
93

156

205

181

180

125 
R. v. Tibshelf (Inhabitants of) (1830) 1 B. \& Ad. 190

Till v. Till [1974] Q.B. 558 C.A.

Timmins v. Timmins [1953] 1 W.L.R. 757; 2 All E.R. 187 C.A.

In re Timmis [1902] 1 Ch. 176

Tinker v. Tinker [1970] P. 136 C.A. .

Reg. v. Tolson (1889) 23 Q.B.D. 168 .

Torok v. Torok [1973] 1 W.L.R. 1066; 3 All E.R. $101 \quad$. 133, 141, 142

Trippas v. Trippas [1973] Fam. 134

$.132,173$

Tulip v. Tulip [1951] P. 378 C.A. $\quad . \quad 76$

Tumath v. Tumath [1970] P. 78, C.A.

Turczak v. Turczak [1970] P. 198

In re Turner (A Bankrupt) [1974]

1 W.L.R. 1556; 1 All E.R. 5 .90, 171

Tymoszczuk v. Tymoszczuk (1964) 108 S.J. 676; The Times 30.6.64 .

Valier v. Valier (1925) 133 L.T. 830.

Vaughan v. Vaughan [1953] 1 Q.B. 762. C.A.

Vaughan v. Vaughan [1973] 1 W.L.R. 1159; 3 All E.R. 449 C.A.

Voller v. Dairy Produce Packers [1962] 1 W.L.R. 960; 3 All E.R. 938

Von Mehren v. Von Mehren [1970] 1 W.L.R. 56; 1 All E.R. 153, C.A.

In re W. [1963] Ch. 556

In re $W$, The Times 28.172

In re $W$. decd. The Times 28.4.75

In re W. (An Infant) [1971] A.C. 682

W. v. W. [1964] P. 67 C.A.

219

$W$. v. $W$. (Financial Provision: Lump Sum) [1976] Fam. 107

$W$. v. W. The Times 26.11.76 . . 205, 208

W. v. W. \& C. [1968] 1 W.L.R. 1310; 3 All E.R. 408 C.A.

W. (R.J.) v. W. (S.J.) [1972] Fam. 152

91

46

189

ichtel v. Wachtel [1973] Fam. 72 C.A $11,79,125,132$, $136,163,172,173-7$, $180,181,184,188$

R. v. Ward (1762) 1 Wm. Bl. 386

211

Warr v. Warr [1975] Fam. 25 .

127

In re Watkins [1953] 1 W.L.R. 1323;

2 All E.R. 1113

24,160

Watts v. Spence [1976] Ch. 165

Watts v. Waller [1973] 1 Q.B. 153 C.A.
Weisz v. Weisz, The Times 16.12 .75 C.A.

Wellesley v. Beaufort (1827) 2 Russ. 1

In re Wernher [1918] 2 Ch. 82 C.A. .

Reg. v. West London Supplementary Benefits Appeal Tribunal ex parte Clarke [1975] 1 W.L.R. 13963 All E.R. 513

Reg. v. West London S.B. Appeal Tribunal ex parte Taylor [1975] 1 W.L.R. 1048; 2 All E.R. 790 .

West v. West, The Times 16.11.73

In re Whittall [1973] 1 W.L.R. 1027; 3 All E.R. 35 .

Whitton v. Garner [1965] 1 W.L.R. 313; 1 All E.R. 70

page

177

209

101

Wild v. Wild [1969] P. 33

Wilkins v. Wilkins [1969] 1 W.L.R. 922; 2 All E.R. 463.

Williams (J.W.) v. Williams (M.A.) [1976] Ch. 278

Williams (L.A.) v. Williams (E.M.) [1974] Fam. 55

223

128

100

186

172

187

222

Wilson v. Wilson [1973] 1 W.L.R. 555; 2 All E.R. 17 C.A.

128

Wilson v. Wilson [1976] Fam. 142 C.A.

In re de Wilton [1900] 2 Ch. 481 .

Winter v. Winter, The Times 14.11 .72

Wolfenden v. Wolfenden [1946] P. 61 .

Wright v. Wright [1976] Fam. 114

Wright v. Wright (Practice Note) [1973] 1 W.L.R. 1145

Wroth v. Tyler [1974] Ch. 30 .

128,134

$R$. v. Wroxton (1833) $4 B$. and $A d$. 640

Wynn v. Wynn [1964] 108 S.J. 239

In re $X$. [1975] Fam. 47 C.A. .

In re Y. (A Minor) (Child in Care: Access) [1976] Fam. 125 C.A. .210, 253

Y. v. Y. (Child: Surname) [1973] Fam. 147

184

40

193

50

131

191

31

218

210

In re Yerburgh [1928] W N 208 • 58

Young v. Young [1964] P. 152. 126

Youngs v. Youngs [1940] 1 K.B. 760. 88

Zamet v. Hyman [1961] 1 W.L.R. 1442 C.A.

AUSTRALIAN CASES

Curran v. Young, 112 (1965) 
Jacombe v. Jacombe [1960] N.S.W.R. 703 and 707

\section{U.S.A. CASES}

In re Alexander (1968) 206 So. 2d. 452 Fla.

Forbush v. Wallace (1972) U.S. 92, S.Ct. 1197

In re Gault (1967) 387 U.S. 1 .
231

page 30 56 244
In re Jewish Child Care Association (Laura Neuberger) (1959) 5 N.Y. 2d. 222; 183 N.Y.S. 2d. $65 ; 156$ N.E. 2 d. 700

State ex rel. Krupa v. Green (1961) 114 Ohio App. 497.

page 231 56 Loving v. Virginia (1969) U.S. 1, 87 S.Ct. 1817 . . . 3 Morgan v. Morgan (1963) $34 \mathrm{Cal}$. Rptr. 82 People ex rel. Rago v. Lipsky (1945) 327 Ill. App. 63 